The abstracts are only available online, free of charge, under www.karger.com/doi/10.1159/000202073

\title{
14th International \\ Conference on Continuous Renal Replacement Therapies (CRRT)
}

February 25-28, 2009, San Diego, Calif.

\section{Abstracts}

Editor

R.L. Mehta, San Diego, Calif.

\section{Contents}

Patient Characteristics

Abstracts 1-22

Emerging Concepts

Abstracts 23-32

Technique Characteristics

Abstracts 33-44

Targeted Interventions

Abstracts 45-54

Future Trends

Abstracts 55-60

CRRT Research

Abstracts 61-69

Author Index 


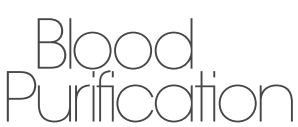

\section{Patient Characteristics}

1

\section{Clinical Utility of Continuous Renal Replacement Therapy in Critically III Patients in Critical Care Unit}

Hong Xiao, Li Peng

Department of Nephrology, Kiang Wu Hospital, Macau, China

Objective: Retrospectively analyze the clinical utility of Continuous Renal Replacement Therapy (CRRT) in Critical Care Unit of Internal Medicine Department.

Method: During the period of Jun 2004 to Dec 2007, CRRT were performed on 42 cases of critically ill patients in our department. General data including age, gender, primary underlying disease, comorbidity, and laboratory examinations pre- and post-treatment were collected and analyzed.

Result: 25 out of 42 patients had been survival from the acute phase of the illness. The survival rate was $59.62 \% .17$ patients died during the acute phase. The death rate was $40.48 \%$. The mean age of the dead group is $74.2 \pm 18.5$ yrs, which was obviously older than that of the survival group (64.7 $\pm 18.7 \mathrm{yrs})$, but there was no statistical significance $=0.497$.

Conclusion: CRRT might provide an effective organ support to the critically ill patients. CRRT might be of help to increase the survival rate of the critically ill patients in the internal medical department.

\section{2}

\section{The Use of CRRT-CVVH in Hematooncological Intensive Care Unit - Single Center Experience}

M. Navratil, Z. Koristek, M. Tomiska, L. Smardova, F. Folber

Department of Internal Medicine-Hematooncology,

Masaryk University Hospital, Brno, Czech Republic

Introduction: The complex care in hematooncological ICU requires sometimes the use of CRRT. As the hematooncological patients have some specifications we retrospectively followed up the results of our effort. As a standard we perform on our ICU CVVH with post dilution on Fresenius Multifiltrate machine.

Patients: From 1.1.2007 to 30.10 .2008 we performed 124 procedures $(1$ procedure $=24 \mathrm{hrs})$ in 31 pts $($ septic shock + MSOF -13 , $\mathrm{VOD}+\mathrm{MSOF}-7$, myeloma $+\mathrm{AKI}-1$, primary amyloidosis + neph- rotic syndrome - 1 , tumor lysis syndrome -4 , hepatorenal failure as a manifestation of basic disease - 2, hepatorenal failure after chemotherapy -2 , TTP + AKI -1 . Anticoagulation - LMWH or defibrotid (in VOD pts) or without anticoagulation.

Results: In 20 from 31 pts the introduction of CVVH in therapeutic process dramatically improved the pts condition and CVVH was the crucial part of intensive care. In 7 pts the level of MSOF with metabolic breakdown was irreversible. In 4 pts the CVVH improved the pts condition but subsequent other complications were the cause of the death. From these 11 pts -5 with refractory sepsis, 2 extreme obese with refractory basic disease, 1 with leukostasis and cerebral hemorrhage, 1 with primary amyloidosis + very low heart left ventricle ejection fraction, 2 with sepsis after salvage chemotherapy and progressive basic disease.

Conclusions: CVVH is integral part in intensive care in hematooncology and the use of this therapeutic modality can significantly improve the results of our therapeutic effort.

\section{3}

\section{Hemodialysis Complications in the Developing World: Iraq Experience}

\section{A.J. Al Mosawi}

Departments of Pediatrics and Continuing Medical Education, University Hospital in Al Kadhimiyia, Baghdad, Iraq

Purpose of the Study: In most areas of the industrialized world, the most common complications during hemodialysis (HD), in descending order of frequency are hypotension, cramps, and nausea and vomiting. The aim of this paper is to report the differing incidence of HD complications.

Methods: During the period from February 2007 to October 2007, 50 patients (18 males, 22 females) with renal failure (RF); $82 \%$ with chronic renal failure (CRF), and $18 \%$ with acute renal failure (ARF) undergone 400 hemodialysis sessions [3-4 hours,2-3 sessions/ week for patients with CRF].The age of the patients ranged from 5 to 7 years; $2 \%$ of the patients under 10 years, $12 \%$ ( $11-20$ years), $14 \%$ (21-30years), $20 \%$ (31-40 years), $28 \%$ (41-50\%), 18\%(51-60years), $6 \%$ (61-70 years).RF was caused by diabetes in $28 \%$ of the patients, hypertension in $22 \%$,glomerulonephritis in $14 \%$,obstructive uropathy in $16 \%$, shock in $8 \%$, and other causes in the remaining $12 \%$ including SLE in 1 patient,HUS in 1 patient, Alport syndrome in 1 patient, pyelonephritis in 1 patient and the cause was undetermined in 1 patient. The Dialysis apparatus used was Gambro with polyflux dialyzer membrane and 1.4-2.1 $\mathrm{m}^{2}$ effective surface area and the flow rate was 200-300 ml/minute. Dialysate composition: Sodium $133 \mathrm{mmol} / \mathrm{L}$, Chloride $97 \mathrm{mmol} / \mathrm{L}$, calcium $1.5 \mathrm{mmol} / \mathrm{L}$, potassium $1.5 \mathrm{mmol} / \mathrm{L}$, magnesium $0.8 \mathrm{mmol} / \mathrm{L}$, acetate $40 \mathrm{mmol} / \mathrm{L}$, glucose $2.1 \mathrm{~g} / \mathrm{L}$.

\begin{tabular}{ll}
\hline KARGER & (c) 2009 S. Karger AG, Basel \\
0253-5068/09/0273-0272\$26.00/0 \\
$\begin{array}{l}\text { Fax +4161306 1234 } \\
\begin{array}{l}\text { E-Mail karger@karger.ch } \\
\text { www.karger.com }\end{array}\end{array}$ & $\begin{array}{l}\text { Accessible online at: } \\
\text { www.karger.com/bpu }\end{array}$
\end{tabular}


Results: Temporary catheter malfunction in 24 patients $(48 \%)$, hypotension in 16 patients (32\%), nausea in 14 patients (28\%), infective hepatitis B in 12 patients $(24 \%)$, itching in 11 patients $(22 \%)$, muscle cramps in 10 patients $(20 \%)$, vomiting in 8 patients $(16 \%)$, headache in 8 patients $(16 \%)$, backache in 7 patients $(14 \%)$, chest pain in 7 patients $(14 \%)$, fever in 6 patients $(12 \%)$, chills in 5 patients $(10 \%)$, fainting in 4 patients $(8 \%)$, disequilibrium in 2 patients $(4 \%)$, seizures in 1 patient $(2 \%)$.

Conclusion: HD complications in our center differ from many previous reports because of the higher incidence of temporary catheter malfunction attributed to delay in the creation of permanent vascular access and the high frequency of hepatitis B infection.

\section{4}

\section{Successful Treatment of Rhabdomyolysis following Cardioversion and Cardiopulmonary Resuscitation with Continuous Venovenous Hemodiafiltration (CVVHDF)}

\author{
J.M. Park', T. Ui Lee ${ }^{2}$, G.R. Chon', J. Ho Wang ${ }^{3}$, H.J. Shin', \\ Y. Chil Choi ${ }^{4}$ \\ ${ }^{1}$ Department of Pediatrics, ${ }^{2}$ Department of Surgery, \\ ${ }^{3}$ Department of Internal Medicine, ${ }^{4}$ Department of \\ Radiology, Konkuk University College of Medicine, \\ Chungju, Chungbuk, Korea
}

A 5-year-old boy was referred to our emergency room because of severe dehydration due to vomiting and diarrhoea over the prior 3 days, and because of comatose mentality which began just prior to visiting emergency room and was worsening. A few minutes after he arrived in our emergency room, his heart rate became flat, and we initiated cardiac massage. After 5 cardioversions, ventricular fibrillation was successfully converted to sinus rhythm. Rhabdomyolysis ensued with elevated of serum creatine kinase ( CK : 19,000 U/L), lactate dehydrogenase (LDH : 7,480 U/L) and myoglobinuria with acute renal failure, which required hemodialysis. Initial blood urea nitrogen $(\mathrm{BUN})$ and creatinine $(\mathrm{Cr})$ concentrations were $90 \mathrm{mg} / \mathrm{dL}$ and 6.5 $\mathrm{mg} / \mathrm{dL}$, respectively. We established a diagnosis of rhabdomyolysis, acute renal failure, multiple organs dysfunction syndrome (MODS), sepsis, pneumonia, disseminated intravascular coagulation, and brain hypoxic damage. The patient was infused with isotonic crystalloid solution preserving a urine output, following which BUN and $\mathrm{Cr}$ reached concentrations of $35 \mathrm{mg} / \mathrm{dL}$ and $3.5 \mathrm{mg} / \mathrm{dL}$, respectively but did not decrease further. There were two episodes of respiratory arrest due to weaning failure after admission to the intensive care unit. We attempted to intermittent hemodialysis 4 times, but the oliguria continued and there was no change in BUN or Cr. The patient's urine output was less than $100 \mathrm{cc} /$ day per day and he became severely edematose and experienced a weight gain of $7 \mathrm{~kg}$, so we initiated continuous venovenous hemodiafiltration (CVVHDF, Prismaflex, Gambro) on the seventh day after admission with his PRISM III score standing at 17. After the CVVHDF for 12 days, the patient was urinating well and his BUN and Cr had returned to normal, as had concentrations of his other liver enzymes, CK, and LDH. One months after initial admission, the patient had recovered and exhibited stable vital signs, and he was discharged from the intensive care unit and is on rehabilitation therapy.

5

\section{Acute Kidney Injury (AKI) and Dialysis Predicts Are Independent Predictors of Mortality in Neonatal Non-Cardiac Patients Receiving Extra-Corporeal Life Support}

\author{
D.J. Askenazi ${ }^{1}$, K. Hamilton², G. Cutter ${ }^{3}$, D. Laney4, \\ R.A. Dimmitt ${ }^{5}$, N. Ambalavanan ${ }^{5}$ \\ ${ }^{1} U A B$ Pediatric Nephrology, ${ }^{2}$ UAB Pediatric Biostatistics, \\ ${ }^{3} \mathrm{UAB}$ Biostatistics, ${ }^{4} \mathrm{CHS}$ ECLS Coordinator, ${ }^{5} \mathrm{UAB}$ Pediatric \\ Neonatology, Birmingham, AL, USA
}

Introduction: Acute kidney injury (AKI) independently predicts mortality in different populations of pediatric and adult critically ill patients, yet these analyses have not been performed in the neonatal populations. Several single center studies in neonates who received extracorporeal life support (ECLS) suggested that AKI portends mortality but small sample size limited the ability to control for confounding variables known to predict poor outcomes.

Methods: The Extracorporeal Life Support Organization registry is a multinational registry that collects data on the majority of all ECLS patients around the world. We performed an analysis of the 11,332 non-cardiac neonatal (0-30 days old at time of ECLS initiation) between 1995 and 2008. Surrogate for AKI used a categorical complication code if a patient had a serum creatinine $>1.5 \mathrm{mg} / \mathrm{dl}$ or if ICD9 for acute renal failure coded during ECLS. Renal replacement therapy (RRT) was coded as a complication and/or CPT coding on the ELSO registry. We performed a logistic stepwise regression analysis to study the effects of AKI and dialysis controlling for demographics, co-morbidities, complications, procedures, underlying diagnosis, $\%$ of RRT in center/ year, year of ECLS, and centers with many $(\geq 10)$ vs. few $(<10)$ procedures/year.

Results: Those with AKI $[\mathrm{N}=238(7.7 \%)]$ had higher mortality rates vs. those without AKI $[\mathrm{N}=66(0.8 \%)] \mathrm{p}<0.0001$. Those who required $\mathrm{RRT}[\mathrm{N}=1146(36.9 \%)]$ had higher mortality rates than those without RRT $[\mathrm{N}=1222(14.9 \%)] \mathrm{p}<0.0001$.

Conclusions: AKI and dialysis are independent predictors of mortality in neonates who require ECLS after adjusting for known predictors of mortality. Ascertainment of AKI risk factors, testing

Table Neonatal Mortality given AKI/ RRT*

\begin{tabular}{llll}
\hline & & OR $(95 \% \mathrm{CI})$ & p-value \\
\hline \multirow{2}{*}{ AKI } & Crude & $10.2(7.7,13.5)$ & $<0.0001$ \\
\multirow{2}{*}{ RRT } & Adjusted & $4.1(2.7,6.1)$ & $<0.0001$ \\
& Crude & $3.3(3.0,3.7)$ & $<0.0001$ \\
& Adjusted & $2.6(2.2,3.0)$ & $<0.0001$ \\
\hline
\end{tabular}

*adjusted for age, birthweight, FiO2@0 \& 24 hrs, handbagging, hours on ecmo, sex, ventilator type, duration on vent pre-ECLS, high or low volume center, mode of ECLS, before vs. after 2000, pneumothorax, pulmonary hemorrhage, liver failure, presence of infections, cardiopulmonary arrest, $\mathrm{pH}<7.2$, brain disease, seizure, \% RRT /center/year, meconium aspiration, congenital diaphragmatic hernia and persistent pulmonary hypertension 
novel therapies and optimizing the timing/ delivery of dialysis may have tremendous impact on survival of non-cardiac neonates who require ECLS.

\section{6}

\section{Acute Kidney Injury (AKI) and Dialysis Predicts Are Independent Predictors of Mortality in Pediatric Non-Cardiac Patients Requiring Extra-Corporeal Life Support (ECLS)}

\author{
D.J. Askenazi ${ }^{1}$, K. Hamilton², G. Cutter ${ }^{3}$, D. Laney4, \\ R.A. Dimmitt ${ }^{5}$, N. Ambalavanan ${ }^{5}$
}

${ }^{1} U A B$ Pediatric Nephrology, ${ }^{2}$ UAB Pediatric Biostatistics, ${ }^{3} \mathrm{UAB}$ Biostatistics, ${ }^{4} \mathrm{CHS}$ ECLS Coordinator, ${ }^{5} \mathrm{UAB}$ Pediatric Neonatology, Birmingham, AL, USA

Introduction: Acute kidney injury (AKI) independently predicts mortality in pediatric and adult critically ill patients but few studies describe this association in pediatric patients who receive extracorporeal life support (ECLS). Several small studies in children requiring ECLS suggests that AKI portends mortality but small sample size limited the ability to control for confounding variables known to predict poor outcomes.

Methods: The Extracorporeal Life Support Organization registry is a multinational registry that collects data on the majority of all ECLS patients around the world. We performed an analysis of the non-cardiac children (30 days to 18 years old at time of ECLS initiation) between 1995 and 2008. AKI was determined by categorical complication if a patient had a serum creatinine $>1.5 \mathrm{mg} / \mathrm{dl}$ or ICD9 Coding for renal failure at any point during ECLS. Renal replacement therapy (RRT) was coded as a complication and/or CPT coding on the ELSO registry. We performed a logistic stepwise regression analysis to study the effects of AKI and dialysis controlling for demographics, co-morbidities, complications, procedures, underlying diagnosis, $\%$ of RRT/center/year, year of ECLS, and centers with many $(\geq 10)$ vs. few $(<10)$ procedures/year.

Results: Those with AKI $[\mathrm{N}=179(15.2 \%)]$ had higher mortality rates vs. those without AKI $[\mathrm{N}=86(5.6 \%)] \mathrm{p}<0.0001$. Those who required RRT $[\mathrm{N}=643(54.7 \%)]$ had higher mortality rates than those without RRT $[\mathrm{N}=425(27.6 \%)] \mathrm{p}<0.0001$.

Conclusions: AKI and RRT are independent predictors of mortality in pediatric patients who require ECLS after adjusting for known predictors of poor outcome. Better understanding of this relationship and determination of the timing of dialysis in ECLS may have tremendous impact on survival. It is important to ascertain the determinants of AKI, study novel early biomarkers and identify the ideal timing of dialysis in pediatric non-cardiac ECLS.

\section{7}

\section{Acute Kidney Injury (AKI) and Dialysis Predicts Are Independent Predictors of Mortality in Adult Non-Cardiac Patients Requiring Extra-Corporeal Life Support (ECLS)}

\author{
D.J. Askenazi , R.L. Mehta², K. Hamilton ${ }^{3}$, G. Cutter ${ }^{3}$ \\ D. Laney ${ }^{4}$, R.A. Dimmitt ${ }^{5}$ \\ ${ }^{1}$ UAB Pediatric Nephrology, ${ }^{2}$ Department of Nephrology, \\ University of California at San Diego, ${ }^{3}$ UAB Biostatistics, \\ ${ }^{4}$ CHS ECLS Coordinator, ${ }^{5}$ UAB Pediatric Neonatology, \\ Birmingham, AL, USA
}

Introduction: Acute kidney injury (AKI) independently predicts mortality in adult critically ill patients but few studies describe this association in those who receive extracorporeal life support (ECLS). Several small studies in the adult ECLS population suggest that AKI portends mortality but small sample size limited the ability to control for confounding variables known to predict poor outcomes.

Methods: The Extracorporeal Life Support Organization registry is a multinational registry that collects data on the majority of all ECLS patients around the world. We analyzed 1348 non-cardiac adult ( $>18$ years old at time of ECLS initiation) enrolled in this registry between 1995 and 2008. AKI was determined by categorical complication if a patient had a serum creatinine $>1.5 \mathrm{mg} / \mathrm{dl}$ or ICD9 Coding for renal failure at any point during ECLS. Renal replacement therapy (RRT) was coded as a complication and/or CPT coding on the ELSO registry. We performed a logistic stepwise regression analysis to study the effects of AKI and dialysis controlling for demographics, co-morbidities, complications, procedures, underlying diagnosis, percentage of patients requiring dialysis, year of ECLS, and centers with many $(\geq 10)$ vs. few $(<10)$ procedures/year.

Results: Those with AKI $[\mathrm{N}=110(16.7 \%)]$ had higher mortality rates vs. those without AKI $[\mathrm{N}=64(9.3 \%)] \mathrm{p}<0.0001$. Those who required RRT $[\mathrm{N}=384(58.4 \%)]$ had higher mortality rates than those without RRT [N=254 (36.8\%)] $\mathrm{p}<0.0001$.

Conclusions: After adjusting for known predictors of poor outcome, RRT requirement remains a strong independent predictor of mortality in adults who require ECLS. Better understanding of this relationship and determination of the timing of dialysis in ECLS may have tremendous impact on survival. Although AKI predicted mortality, no difference in survival was seen in those after confounder adjustment. This effect may have been due to ascertainment bias

Table Adult Mortality given AKI/ RRT

\begin{tabular}{llll}
\hline & & OR $(95 \% \mathrm{CI})$ & p-value \\
\hline \multirow{2}{*}{ AKI } & Crude & $2.0(1.4,2.7)$ & $<0.0001$ \\
& Adjusted & $1.0(0.6,1.7)$ & $=0.94$ \\
RRT & Crude & $2.4(1.9,3.0)$ & $<0.0001$ \\
& Adjusted & $2.4(1.7,3.5)$ & $<0.0001$
\end{tabular}

*adjusted for age, birth weight,FiO2@0 \& 24 hrs, hand bagging, hours on ecmo, sex, ventilator type, duration on vent pre-ECLS, high or low volume center, mode of ECLS, before vs. after 2000, pneumothorax, pulmonary hemorrhage, liver failure, presence of infections, cardiopulmonary arrest, $\mathrm{pH}<7.2$, brain disease, seizure, and \% RRT /center/year. 
given the imprecise method of defining AKI. Outcomes in this vulnerable population may be improved by better understanding risk factors, exploring therapies and testing strategies to optimally dose and initiate dialysis.

\section{8}

Prolonged Intubation Greater than 2 Weeks in Pediatric Patients Receiving Continuous Renal Replacement Therapy (CRRT) Portends Extremely Poor Prognosis

\section{A.N. Chua, D. Askenazi, R. Hackbarth, F.X. Flores, \\ S.L. Goldstein \\ Prospective Pediatric Continuous Renal Replacement Therapy (ppCRRT) Registry Group, Houston, TX, USA}

Continuous renal replacement therapy (CRRT) is widely used in critically ill pediatric patients with acute kidney injury (AKI). Several studies have evaluated variables at CRRT initiation as predictors of survival, and showed CRRT initiation at lesser degrees of fluid overload were associated with improved survival. No studies exist, however, to provide prognostic information based on variables during the CRRT course. Data from the Prospective Pediatric Continuous Renal Replacement Therapy (ppCRRT) Registry were reviewed to identify predictors of non-survival at various times of CRRT duration (2 days, 8 days, 15 days, 22 days, and $>28$ days). Subjects were included if CRRT duration was greater than or equal to 2 days, and if complete mean airway pressure (Paw) and pressor data were available. Variables evaluated were persistently compromised respiratory status as measured by increase or no change in mean airway pressure and continued pressor support. Total number of patients with \%survival in parentheses at various times of CRRT duration are as follows: 2 days, 185 pts (51\%); 8 days, 94 pts (46\%); 15 days, 41 pts (41\%); 22 days, 21 pts (38\%); greater than 28 days, 10 pts $(50 \%)$.

At greater than or equal to 15 days, $100 \%$ of patients without improvement in Paw died as demonstrated in the table below.

Our ppCRRT data suggest that persistence of multi-organ dysfunction after 2 weeks of CRRT portends poor prognosis for survival. This information may be useful to provide prognostic assistance to clinicians during the course of CRRT. Larger studies however are warranted to confirm these findings prior to making clinical decisions regarding withdrawal of care.

\section{9}

\section{Continuous Renal Replacement Therapy in Intensive Care Unit - Single Center Experience}

\author{
H. Kim, J. Kim, E. Hwang, M. Chang, S. Han, S. Park \\ Department of Nephrology, Keimyung University, Daegu, \\ South Korea
}

The mortality rate in critically ill patients with AKI remain unacceptably high, despite numerous advances in dialysis techniques and intensive care medicine during the last few decades. We evaluated clinical characteristics, disease severity, mortality, and prognostic factors in ICU patients with AKI requiring CRRT.

Methods: We retrospectively reviewed the medical records of all ICU patients who received CRRT at Keimyung university hospital from September 2002 to October 2007. We identified demographic variables, underlying diagnosis, clinical features, characteristics of CRRT, mortality, prognostic factor.

Results: The mean age of patients was 58.3 years. The time from admission to initiation of CRRT was $63.5 \pm 40.7$ hours. The mechanical ventilation rate was $82.8 \%$, vasoactive drug $79.3 \%$ and sepsis $40 \%$. APACHE II score was $25.2 \pm 7.9$, SAPS II 48.1 \pm 15.1 , CCF score $9.3 \pm 3.6$, the numbers of organ dysfunction was $2.1 \pm 1.3$. Overall mortality rate was $51 \%$. Compared with sepsis and nonsepsis group, the number of organ dysfunction and severity of illness were significantly higher in sepsis patients than that of nonsepsis patients. A mortality rate of sepsis patients was significantly higher than nonsepsis patients. In our study, risk factors of mortality were the number of organ dysfunction, severity of illness, MAP, platelet count, and serum albumin level. All these factors were the significant risk factors for mortality in univariate analysis, however their statistical significance were lost in multiple linear regression analysis.

Conclusions: A large scaled, prospective randomized multicenter trials needed to confirm the beneficial effect of CRRT in patient with ARF in ICU.

Table (for Abstract 8) \% Non-survival in Patients Based on Respiratory Status and Pressor Support throughout Course of CRRT Duration.

\begin{tabular}{lll}
\hline $\begin{array}{l}\text { Timepoint of CRRT } \\
\text { Duration }\end{array}$ & $\begin{array}{l}\text { \% Non-survival when still } \\
\text { intubated without respiratory } \\
\text { status improvement }\end{array}$ & $\begin{array}{l}\% \text { Non-survival in those with } \\
\text { continued pressor support }\end{array}$ \\
\hline 2 days & $76 \%(n=76)$ & $71 \%(n=87)$ \\
8 days & $86 \%(n=36)$ & $75 \%(n=40)$ \\
15 days & $100 \%(n=15)$ & $74 \%(n=18)$ \\
22 days & $100 \%(n=6)$ & $78 \%(n=9)$ \\
greater than 28 days & $100 \%(n=3)$ & $75 \%(n=4)$ \\
\hline
\end{tabular}

14th International Conference on Continuous 
10

\section{Is the Profile of AKI Needing Intensive Care Changing in Developing Countries}

\author{
S. Samavedam¹, R. Chakravarty², V. Reddy², \\ Harikrishna ${ }^{2}$, Mallikarjun ${ }^{3}$ \\ ${ }^{1}$ Department of Critical Care, ${ }^{2}$ Department of Nephrology, \\ ${ }^{3}$ Department of Internal Medicine, Care Hospitals, \\ Hyderabad, AP, India
}

Background: The outcomes of AKI in developing countries is considered to be better due to the difference in the etiology of the condition. This study aims to examine whether the outcomes of AKI needing intensive care are any different.

Aim: To study the factors involved in the outcome of patients with AKI needing ICU care in a tertiary care centre from southern India.

Material and Methods: All consecutive patients admitted to the Intensive Care Unit of CARE hospitals for management of manifestations of AKI were prospectively studied over a two month period from 1 Sep 2008 to 30 Nov 2008. APACHE II scoring was recorded for all patients. Patients with Multi-Organ Failure (MOF) had a daily SOFA score recorded. Comorbidities and Drug history were recorded. The major outcomes studied were ICU and in hospital mortality. The secondary outcome measured were ICU days, Hospital days, Ventilator days, RRT days and discharge destination. Odds Ratios were calculated for the different variables and parameters.

Results: A total of 30 patients with a label of AKI were managed during the study period. This was about $20 \%$ of the total ICU admissions. Twenty two of these patients were male. The mean APACHE II of all patients with AKI was 26. Metabolic acidosis and Respiratory failure were the presenting problems in 15 patients. MOF was recorded at admission in 8 patients. Hypertension and Diabetes were recorded among 20 and 15 patients respectively. Ischemic Heart Disease complicated by AKI was the problem among 11 patients and 10 patients had CKD. A total of 14 patients survived. The survivors stayed for 12 days in the ICU compared to 6.5 days by the non-survivors. All non survivors needed RRT while 10 patients out of 14 survivors needed RRT. Nine out of 11 patients with IHD did not survive.

Conclusions: AKI is seen in about $20 \%$ of tertiary care ICU admissions.

AKI is a component in $25 \%$ of MOF patients.

Mortality in MOF is higher if AKI is a component

IHD complicated by AKI has a 7.0 OR for mortality

Preexistent CKD does not have an adverse effect on outcome of AKI

The profile of AKI in tertiary care ICUs and the mortality rates do not seem to be much different between the developed countries and India

\begin{tabular}{llll}
\hline Comorbidity & Survivors & Non survivors & Odds ratio \\
\hline DM & 8 & 7 & 1.4 \\
Hypertension & 10 & 10 & 1.5 \\
Ischemic Heart Disease & 2 & 9 & 7 \\
Hypotension & 3 & 7 & 4.2 \\
CKD & 9 & 1 & 0.3 \\
CHF & 3 & 4 & 1.1 \\
\hline
\end{tabular}

11

\section{Therapeutic Plasma Exchange (TPE) for Recurrent Clotting During Continuous Renal Replacement Therapy (CRRT)}

\author{
A. Cosmin, L. Juncos, T. Fulop \\ Department of Medicine/Nephrology, University of MS \\ Medical Center, Jackson, MS, USA
}

Introduction: Clotting of the extracorporal circuit (ECC) can be a recurrent problem on CRRT. It is most often attributed to access and filter characteristics or insufficient anticoagulation. Patient-specific characteristics, such us acquired elevation of clotting factors, are less frequently considered in the differential.

Presentation of the Case. A 17 year old otherwise healthy white male was admitted to Intensive Care Unit following a motor vehicle accident with multiple fractures. His hospital course was complicated by aspiration pneumonia, ARDS with refractory hypoxemia, hypotension, liver dysfunction and oliguric acute kidney injury. He was consequently placed on CRRT using an AN69 membrane and regional citrate anticoagulation. However, repeated episodes of ECC clotting occurred with the average filter life being approximately 3.5 hours. Adjusting citrate rates, adding low-dose heparin to the circuit and exchanging the dialysis catheter did not help. Trials of intermittent hemodialysis using heparin were also prematurely terminated due to ECC clotting. Consequently, we were unable to deliver even the minimal acceptable doses of renal replacement therapy. A subsequent evaluation for a primary hypercoagulable state was negative, but his fibrinogen was very elevated $(1320 \mathrm{mg} / \mathrm{dl})$, likely induced by his severe inflammatory state. We therefore considered that the elevated fibrinogen was causing the frequent clotting/clogging of the ECC. We therefore tested whether total plasma exchange (TPE), which removes fibrinogen (and other acute phase reactant molecules), would decrease ECC clotting. A single-session TPE was performed in which we replaced $4006 \mathrm{ml}$ of 'dark golden' plasma with 2 liters of $5 \%$ albumin and 1750 liters of normal saline (1.1 times his estimated plasma volume). This resulted in an acute drop in fibrinogen to 615 $\mathrm{mg} / \mathrm{dl}$. Despite fibrinogen levels rebounding the next day to 827 , no further episodes of premature ECC clotting occurred, suggesting that the fibrinogen, or perhaps another plasma factor removed by TPE, was responsible for premature ECC clotting.

Conclusions: Markedly elevated fibrinogen, or similar acute phase reactants, may cause recurrent premature clotting of extracorporeal circuits. Decreasing the circulating levels of these factors with TPE may help decrease the 'hypercoagulable state' and consequently improve delivery of dialysis and reduce filter and blood consumption. 


\section{2 Continuous Renal Replacement Therapy: A
Survey of Practice in an Intensive Care Unit}

M. Seo, A. Choi, J. Suh, Y. Cho, Y. Sung

Department of Intensive Care Unit, Samsung Medical

Center, Seoul, Korea

Objectives: For future reference, Continuous Renal Replacement Therapy (CRRT) treatment effect and operation status for acute renal failure patients have been investigated by 1) checking patients' status undergoing CRRT, 2) understanding clinical properties related to CRRT, and 3) grasping the relation between CRRT's properties and the mortality during patient discharge.

Method: CRRT assessment sheet and situation sheet were developed for ourselves and two expert nurses have investigated 731 patients undergoing CRRT in an intensive care unit from Jan/2002 to Dec/2006

Results: In the survey, the total number of CRRT trials is 713 . CRRT treatment has been increased since 2002, when there were no CRRT expert nurses. In 2006, the number of CRRT treatment has been increased to 194. Hematooncology is ranked the first among medical departments where give CRRT treatment (20.1\%). General surgery is $14.9 \%$, Nephrology is $13.8 \%$, Cardiology is $11.8 \%$, Thoracic surgery is $11.5 \%$ and Pulmonology is $10.8 \%$. The above 6 departments occupy $82.9 \%$ out of $100 \%$. The most common reason of CRRT treatment is azotemia $(40.0 \%)$. The second reason is volume overload $(34.9 \%)$, the third one is metabolic anomaly $(13.3 \%)$, the fourth one is electrolyte imbalance $(8.6 \%)$ and the next one is toxin removal $(3.3 \%)$ However, electrolyte imbalance decreased while metabolic anomaly and toxin removal increased. Before CRRT treatment, patients have $78.6( \pm 55.5)$ of BUN value and $5.0( \pm 3.2)$ of $\mathrm{Cr}$. value. The standard values of BUN and Cr. have been lowered so that CRRT could be applied in the initial stage. Compared the survival group with the death group, there are differences among the medical departments and the main diagnosis group. In the properties related to CRRT, BUN, Cr. value, APACHE II score, mean blood pressure and oliguria show differences $(<0.05)$ as well.

Conclusion: The survey has been made from a general hospital. Therefore, more surveys from similar hospitals should be made in order to generalize the results. We also propose that a research has to be done how CRRT treatment affects patient's survival/death rate.

\section{3}

\section{CRRT Experiences on Maintenance Hemodialysis Patients}

\author{
J.-H. Kim ${ }^{1}$, W.-D. Lee', J.-K. Kim² \\ Department of Nephrology, ' Good Gang-An Hospital, \\ ${ }^{2}$ Bongseng Hospital, Busan, South Korea
}

The continuous veno-venous hemodiafiltration(CVVHDF) by continuous renal replacement therapy(CRRT) machine have been used acute kidney injury in critically ill patients. But, in hemodynamically unstable maintenance hemodialysis patients, the methods can be applied. We report the temporary use of CRRT in the management of hemodynamically unstable dialysis patients.

The data given in this study relate to the period from 07.01.200811.30.2008. We performed CRRT in 5 patients, who had been maintenance hemodialysis 3times weekly. All patients were treated with CVVHDF, Prismaflex. Hypotension etiology of our patients was as follows:

- 1 patient underwent cardiac arrest due to air embolism during hemodialysis session.

1 patient suffered from cholangitis.

1 patient had sepsis, which is caused by skin infection.

2 patients suffered from severe heart failure.

These all patients needed inotropes due to hypotension. The durations of CVVHDF were 24-72hours. Blood flow rates were 100$140 \mathrm{ml} / \mathrm{min}$. All patients with heart failure were dead, but remained patients were recovered. Especially, the patient with air embolism needed hyperbaric oxygen therapy through ventilator. High peep ventilator therapy lower blood pressure. But, during ventilator therapy, the patient had overcome renal replacement therapy through CVVHDF.

Conclusion: Hemodynamically unstable dialysis patients is difficult to maintain general hemodialysis therapy. But, during hypotensive period, treatment with CVVHDF will provide good bridge dialysis method to them.

14

\section{Severe Systemic Oxalalosis Causing Premature CVVHD Failure after Ethylene Glycol Ingestion}

M.L. Britton, A.S. Podoll, K.W. Finkel

Division of Renal Disease and Hypertension, University of Texas Health Science Center, Houston, TX, USA

We present a case of repeated hemofilter failure during treatment of ethylene glycol poisoning with CVVHD using regional citrate anticoagulation (RCA). A 53 year old man with a history of schizophrenia presented to the MICU after intentional ethylene glycol ingestion. On presentation, physical exam revealed an intubated, unresponsive, hypotensive male. He had severe metabolic acidosis with a $\mathrm{pH}$ less than 6.95 and bicarbonate of $8 \mathrm{mEq} / \mathrm{L}$, creatinine $2.4 \mathrm{mg} / \mathrm{dL}$, potassium $6.4 \mathrm{mEq} / \mathrm{L}$ and oliguria. Osmolality was $323 \mathrm{mOsm} / \mathrm{kg}$ with an osmolar gap of 18.4. Fomepizole was initially started by standard protocol but the dose was adjusted once CVVHD commenced to account for its clearance at a dose of $700 \mathrm{mg}$ intravenously every four hours. CVVHD was started with RCA. Initial post filter ionized calcium was $0.46 \mathrm{mmol} / \mathrm{L}$. The prescribed dose of dialysis was $40 \mathrm{ml} / \mathrm{kg} / \mathrm{hr}$.

Three hours into the treatment the hemofilter clotted and the nursing staff observed palpable, crystalline material in the filter and venous port. Anatomical pathology revealed typical coagulated blood without crystals. CVVHD was restarted with a post filter ionized calcium of $0.2 \mathrm{mmol} / \mathrm{L}$ and citrate was adjusted. Over the first 24 hours, four hemofilters clotted with a mean filter life of 7.3 hours. Prior to each clotting episode similar granulated material was noticed both in the tubing and in the hemofilter. Despite the difficulty encountered with therapy, ethylene glycol levels decreased from $25 \mathrm{mg} / \mathrm{dL}$ to 17 $\mathrm{mg} / \mathrm{dL}$ after 3.5 hours of CVVHD. Serum oxalate levels decreased from $86 \mu \mathrm{M} / \mathrm{L}$ to undetectable levels within 24 hours. No further epi- 
sodes of hemofilter failure were noted after serum ethylene glycol and oxalate levels were undetectable. The dose of dialysis delivered in the first 24 hours was $37.8 \mathrm{ml} / \mathrm{kg} / \mathrm{hr}$. The patient was transitioned to intermittent hemodialysis on hospital day three and renal recovery occurred 9 days after admission.

Although severe cases of systemic oxalosis from ethylene glycol ingestion can effectively be treated with CVVHD, we observed that even with effective RCA, multiple episodes of hemofilter failure can occur from apparent crystalline deposition of oxalate. Future hemofilter analysis by electron microscopy in this case is planned.

15

\section{Is the RIFLE scoring system pertinent to evaluate acute renal failure?}

\section{J.-P. Delabre', L. Amigues'1 , O. Jonquet², B. Canaud ${ }^{1}$, K. Klouche ${ }^{1}$ \\ ${ }^{1} \mathrm{CHU}$ Lapeyronie, Montpellier, France, ${ }^{2} \mathrm{CHU}$ Gui de Chauliac, Montpellier, France}

RIFLE scoring is now an established score for grading acute renal failure (ARF), designed to standardize its definition and severity. However, the analysis of current medical reports on ARF epidemiology showed that its mode of calculation varies thoroughly. Our objective was to assess the grading of ARF of our ICU patients using the different methods of RIFLE score calculation reported in literature and to search for an eventual correlation between each of them.

Methods: A Medline query with 'RIFLE' and 'renal insufficiency, acute' identified methods of RIFLE scoring which differed regarding to the calculation mode.

From December 2008 to November 2009 we applied these methods to our patients suffering from ARF which required a renal replacement therapy during its course. RIFLE scores were retrospectively calculated and colliged daily from admission to the first dialysis day according to each method.

Results: Five methods of RIFLE calculation were found (number 1-5 in table). Differences between these modes of RIFLE calculation were mainly related to the estimation of GFR, appreciation of diuresis (included or not or frequency of diuresis collection) and awareness of former renal function.

Twenty nine patients were included, mean age 60 years $(\mathrm{SD}=16.4)$, IGSII 68 (31-113), sex ratio 6.25H/1F. ARF cause was hemodynamic in 24 patients $(83 \%)$, septic in $21(72 \%)$, toxic in $11(38 \%)$ (multiples causes possible).
We studied 110 days. patients and calculated 110x5 RIFLE scores.

As shown in the table, the prevalence of different stages of ARF varies significantly according to the method of RIFLE score calculation used $(p<0.01)$. Global agreement between methods is moderate $(\mathrm{kappa}=0.49)$. Best agreement was found between methods 1 and 4 (kappa $=0.88)$ and worse agreement between methods 1 and 2 (kappa $=0.22$ ).

Conclusion: The agreement between each method of calculation of RIFLE score is low and seems to be insufficient to lead to an optimal reproducibility. Our results strongly suggests that RIFLE score calculation should be more precise and uniformized otherwise comparisons of ARF groups across studies will induce significant misinterpretations.
16

Improvement in Mortality in Severely Burned Patients with Acute Lung Injury/ Acute Respiratory Distress Syndrome and Acute Kidney Injury with Early Continuous Venovenous Hemofiltration

\author{
J.B. Lundy', K.K. Chung ${ }^{2}$, S.F. Wolf2, D.J. Barillo², B.T. King ${ }^{2}$, \\ C.E. White ${ }^{2}$, L.H. Blackbourne ${ }^{2}$ \\ ${ }^{1}$ Department of Trauma/Surgical Critical Care/Burns, \\ Brooke Army Medical Center, ${ }^{2}$ Institute of Surgical \\ Research, Fort Sam Houston, TX, USA
}

Purpose: To report experience of one Burn Intensive Care Unit (BICU) with early Continuous Venovenous Hemofiltration (CVVH) for Acute Kidney Injury (AKI) in patients with concomitant Acute Lung Injury/Acute Respiratory Distress Syndrome (ALI/ARDS). Findings on our overall experience will be reported elsewhere.

Methods: Since November 2005, we have utilized CVVH in burned patients with AKI with or without circulatory shock. We retrospectively reviewed the characteristics of patients admitted to the BICU with greater than $30 \%$ total body surface area (TBSA) burns. Patients diagnosed with AKI or with nephrology consultation made up the control arm of the study. CVVH treated patients comprised the study arm. Demographics, laboratory, hemodynamic, and oxygenation parameters at baseline were recorded. A subgroup of patients with partial pressure of oxygen to fraction of inspired oxygen ratios (PFR) below $300 \mathrm{mmH}_{2} \mathrm{O}$ was identified to analyze outcomes. Mortality at 28 days and in-hospital were also recorded.

Table for abstract 15

\begin{tabular}{llllll}
\hline Method & 1 & 2 & 3 & 4 & 5 \\
\hline $\begin{array}{l}\text { GFR estimate } \\
\text { Diuresis collection }\end{array}$ & $\begin{array}{l}\text { creatininemia } \\
\text { hourly } \\
\text { Anterior GFR }\end{array}$ & $\begin{array}{l}\text { creatininemia } \\
\text { daily }\end{array}$ & $\begin{array}{l}\text { MDRD } \\
\text { unused }\end{array}$ & $\begin{array}{l}\text { creatininemia } \\
\text { unused }\end{array}$ & $\begin{array}{l}\text { Cockroft } \\
\text { hourly } \\
\text { known }\end{array}$ \\
No ARF & $33(30 \%)$ & $49(45 \%)$ & $18(16 \%)$ & $38(35 \%)$ & $27(24 \%)$ \\
RIFLE-R & $19(17 \%)$ & $11(10 \%)$ & $35(32 \%)$ & $20(18 \%)$ & $25(23 \%)$ \\
RIFLE-I & $20(18 \%)$ & $21(19 \%)$ & $30(27 \%)$ & $17(15 \%)$ & $34(31 \%)$ \\
RIFLE-F & $38(35 \%)$ & $29(26 \%)$ & $27(25 \%)$ & $35(32 \%)$ & $24(22 \%)$ \\
\hline
\end{tabular}


Results: There were 486 patients admitted in the control period and 361 during the study period. A total of 28 patients with AKI were included during the control period. In the study arm 29 patients were treated with CVVH at a mean dose of $57+/-19 \mathrm{ml} / \mathrm{kg} / \mathrm{hr}$ for a mean duration of treatment of $5.6+/-4$ days. The diagnosis of ALI/ARDS was present in $16 \mathrm{CVVH}$ treated patients and 20 control patients $(\mathrm{p}=0.2033)$ with similar baseline PFR $(174+/-78$ versus $186+/-64$; $\mathrm{p}=0.63$ ). Both groups were similar in \%TBSA, full thickness \%TBSA, inhalation injury, injury severity score, and initial BUN and creatinine. In the CVVH patients, a significant improvement in PFR at 24 hours was seen when compared to baseline $(327+/-122$ vs $174+/-78$, $\mathrm{p}<0.001)$. This was not evident in the control group (206+/-131 vs $186+/-64, \mathrm{p}=\mathrm{ns})$. Both 28 -day $(31.3 \%$ vs. $85 \%)$ and in-hospital mortality (44\% vs. $95 \%$ ) were significantly lower in the CVVH arm in with ALI/ARDS (both $\mathrm{p}<0.002$ ).

Conclusion: The early use of CVVH in burn patients with AKI led to an improvement in oxygenation in the subgroup of patients developing ALI/ARDS and was associated with a dramatic reduction in mortality.

\section{7 \\ Pre-existing End Stage Renal Disease Is Associated with Decreased Mortality in Critically III Adults Requiring Continuous Renal Replacement Therapy}

\author{
A.M. Walcher ${ }^{1}$, A. Keniston ${ }^{2}$, P.F. Dennen ${ }^{1,2}$ \\ ${ }^{1}$ Department of Renal Diseases and Hypertension, \\ University of Colorado Denver, Aurora, CO, USA, ${ }^{2}$ Denver \\ Health Medical Center, Denver, CO, USA
}

Purpose: To determine the impact of end stage renal disease (ESRD) on overall hospital mortality in patients requiring continuous renal replacement therapy (CRRT).
Background: Acute kidney injury (AKI) requiring CRRT is associated with a high mortality. Patients with chronic kidney disease (CKD) have a higher overall mortality when compared to patients without CKD. Few studies have reported on the impact of baseline kidney function on hospital mortality in patients requiring CRRT. In a single study, patients with acute on chronic kidney disease that required CRRT had a lower mortality than those with normal baseline kidney function who needed CRRT $(31.3 \%$ vs $83.3 \%, p=0.019)$. To our knowledge, there is no published data exploring the impact of ESRD on hospital mortality in critically ill adults requiring CRRT.

Methods: We performed a retrospective chart review of 210 ICU patients (medical, surgical and trauma) who received CRRT at a single institution between 2002 and 2008. Our primary outcome was hospital mortality. We defined ESRD as chronic dialysis dependence and normal kidney function as an admission serum creatinine ( $\mathrm{SCr}$ ) less than $1 \mathrm{mg} / \mathrm{dL}$. Secondary outcomes included dialysis dependence at hospital discharge and the impact of race, gender, age, admitting service, diabetes, and admission serum albumin on hospital mortality.

Results: Of 210 patients requiring CRRT, 29 had ESRD on admission (13.8\%) and $181(86.2 \%)$ were not dialysis dependent (mean admission SCr $3.1+/-3.1 \mathrm{mg} / \mathrm{dL}$ ). Of 181 patients without ESRD, $30(16.6 \%)$ had a $\mathrm{SCr}<1 \mathrm{mg} / \mathrm{dL}$ on admission. Hospital mortality in ESRD patients was $37.9 \%$ compared to $76.7 \%$ in the group with admission $\mathrm{SCr}<1 \mathrm{mg} / \mathrm{dL}(\mathrm{p}=0.003)$. Hospital mortality was lower in ESRD patients vs. unselected patients without ESRD, although this difference did not reach statistical significance $37.9 \%$ vs $56.4 \%, \mathrm{p}=0.06)$. These results were likely impacted by the large percentage of patients with unknown baseline SCr (44\%). Patients in this cohort with $\mathrm{CKD}$ at baseline could have falsely lowered the mortality rate, thus minimizing the difference between the groups.

There was no overall difference in mortality based on race, gender, age or admission service. Both diabetes and a higher admission serum albumin were associated with a lower hospital mortality ( $41.7 \%$ vs $57 \%, \mathrm{p}=0.04$ and $2.8 \mathrm{~g} / \mathrm{dL}$ vs $2.49 \mathrm{~g} / \mathrm{dL}, \mathrm{p}=0.005)$. Twenty one percent of survivors (excluding those with ESRD on admission and those discharged to hospice) remained dialysis dependent at the time of hospital discharge.

Table for abstract 17

\begin{tabular}{|c|c|c|c|c|c|c|}
\hline & $\begin{array}{l}\text { Mortality } \\
\text { No }(\mathrm{N}=97)\end{array}$ & $\begin{array}{l}\text { Mortality } \\
\text { Yes }(N=113)\end{array}$ & P-value & $\begin{array}{l}\text { ESRD } \\
\text { No }(N=181)\end{array}$ & $\begin{array}{l}\text { ESRD } \\
\text { Yes }(N=29)\end{array}$ & P-value \\
\hline Age (mean +/- SD) & $50+/-13$ & $53+/-15$ & NS & $51+/-14$ & $58+/-12$ & 0.004 \\
\hline Male gender, n (\%) & $73(75 \%)$ & $79(70 \%)$ & NS & $138(76 \%)$ & $14(48 \%)$ & 0.002 \\
\hline Non-white race, n (\%) & $60(62 \%)$ & $58(51 \%)$ & NS & $96(53 \%)$ & $22(76 \%)$ & NS \\
\hline $\begin{array}{l}\text { Baseline creatinine } \\
\text { established, } \mathrm{n}(\%) \\
\text { Serum creatinine (mean } \\
\mathrm{mg} / \mathrm{dL}+/-\mathrm{SD})\end{array}$ & $41(42 \%)$ & $61(54 \%)$ & NA & $102(56 \%)$ & NA & NA \\
\hline Baseline (if established) & $1.2+/-0.7$ & $1.1+/-0.7$ & NS & $1.2+/-0.7$ & NA & NA \\
\hline Admission & $4.9+/-4.3$ & $2.7+/-2.6$ & $<0.0001$ & $3.1+/-3.1$ & $7.7+/-4.4$ & $<0.0001$ \\
\hline $\begin{array}{l}\text { Discharge } \\
\text { Serum albumin (mean } \\
\mathrm{g} / \mathrm{dL}+/-\mathrm{SD})\end{array}$ & $2.6+/-2.2$ & $2.5+/-1.9$ & NS & $2.2+/-1.7$ & $4.6+/-3.2$ & $<0.0001$ \\
\hline Baseline (if established) & $3.6+/-0.6$ & $3.6+/-0.5$ & NS & $3.7+/-0.5$ & $3.2+/-0.3$ & NS \\
\hline Admission & $2.8+/-0.8$ & $2.5+/-0.7$ & 0.005 & $2.6+/-0.8$ & $2.9+/-0.7$ & NS \\
\hline $\begin{array}{l}\text { Discharge } \\
\text { ICU service, n (\%) }\end{array}$ & $2.6+/-0.6$ & $2.2+/-0.8$ & $<0.0001$ & $2.3+/-0.7$ & $2.9+/-0.6$ & 0.0004 \\
\hline Medicine/Cardiology & $71(74 \%)$ & $75(68 \%)$ & NS & $124(70 \%)$ & $22(80 \%)$ & NS \\
\hline General Surgery & $9(9 \%)$ & $15(13 \%)$ & NS & $20(11 \%)$ & $4(14 \%)$ & NS \\
\hline Trauma Surgery & $16(17 \%)$ & $21(19 \%)$ & NS & $34(19 \%)$ & $3(10 \%)$ & NS \\
\hline Diabetic, n (\%) & $42(58 \%)$ & $30(42 \%)$ & 0.0414 & $56(78 \%)$ & $16(22 \%)$ & 0.0298 \\
\hline
\end{tabular}

14th International Conference on Continuous 
Conclusion: Patients with ESRD who require CRRT during a critical illness have lower hospital mortality than patients with normal kidney function on admission. These findings suggest that the acute loss of kidney function may play an active role in critical illness. Objective measurements of inflammatory markers and larger observational studies are needed to confirm these findings. In conclusion, the presence of ESRD, diabetes and higher serum albumin on admission are associated with a decreased mortality in critically ill patients requiring CRRT.

\section{8}

\section{Crush Syndrome Complicating Pulmonary Contusion After Wenchuan Earthquake: A Report of Three Cases and Review of the Literature}

\author{
Tao Ye, Wang Li, Hu Zhangxue, Liu Fang, Fu Ping, \\ Huang Songmin, Wang Youjuan \\ Department of Nephrology, West China Hospital, Sichuan \\ university, Chengdu, China
}

Objective: To assess the characteristics and treatment of patients with crush injury complicating pulmonary contusion after Wenchuan earthquake.

Methods: 2600 patients were transferred to West China Hospital in Chengdu, China after Wenchuan earthquake and 69 cases of crush syndrome with acute kidney injury were treated in the department of nephrology. The medical records of 3 patients complicating pulmonary contusion were retrospectively studied and literature was reviewed.

Results: Three patients were male, at the age of 10, 18 and 53 respectively, who were rescued from the collapsed building 5 hours, 20 hours, 24 hours after the earthquake. They all appeared hematuria, anuresis sequentially and had no respiratory symptoms on admission. After receiving continuous veno-venous hemofiltration $(\mathrm{CVVH})$ or intermittent hemodialysis (IHD), they gradually developed a cough accompanied by hemoptysis and shortness of breath. Pulmonary contusion was diagnosed based on the clinic characteristics and the findings of chest radiograph or computerized tomogram (CT) scan. The following treatments were applied: strengthening the oxygen inhalation, reducing the fluid infusion, increasing the dialysis ultrafiltration, extending the time of dialysis and giving albumin or plasma infusion to improve the colloid osmotic pressure. One of them developed acute respiratory distress syndrome (ARDS), who was successfully retrieved by intubation and mechanical ventilation support. Their renal functions were fully restored, followed the disappearance of respiratory symptoms. Three of them were recovered and discharged from the hospital.

Conclusions: Pulmonary contusion, when coexist with crush syndrome after earthquake is easy to be misdiagnosed. Early diagnosis and intervention is of great importance to prevent from developing to respiratory failure and then multiple organ failure. Ventilator support and CVVH took critical roles in the treatment of these patients.
19

\section{Infections in Crush Syndrome: A Retrospective Observational Study after the Wenchuan Earthquake}

\author{
Chen Xiaolei, Hu Zhangxue, Fu Ping, Tao Ye, Liu Fang, \\ Qin Wei \\ Department of Nephrology, West China Hospital, Sichuan \\ University, Chengdu, China
}

Objectives: To investigate the characteristics of infections in the victims who suffered from crush syndrome after the Wenchuan earthquake.

Methods: Clinical data from patient's files during the time from 13 May to 15 June 2008 were analyzed retrospectively. 58 patients who got complete records were included. Patients' demographic data, physical and laboratory findings, disposition and outcome were involved.

Results: Median age of the patients (35 males and 23 females) was 25.5 years (range 9 83 years). The median duration under the rubble was 20 hours (range $2 \sim 73 \mathrm{~h}$ ). Fasciotomies were performed on 29 patients and hemodialysis were received by 47 patients, thirtyseven of whom treated by continuous renal replacement therapy. Microbial pathogens were detected in the samples from 39 patients $(67.2 \%)$ and twenty-nine of them (50\%) were diagnosed as sepsis. Wound and pulmonary infections were the main presentation of infectious complication. Acinetobacter baumanii, Pseudomonas aeruginosa, Enterobacter cloacae and Escherichia coli were isolated in nineteen, seventeen, thirteen, and nine patients, respectively. The major bacterial isolates from wound infections were Acinetobacter baumanii and Pseudomonas aeruginosa, and those from pulmonary infections were Acinetobacter baumanii and Enterobacter cloacae. Twenty-six of the patients (44.8\%) were influenced by infection after 48 hours after hospitalization. The incidence of infection was higher in the patients who received fasciotomies than those did not $(82.8 \%$ vs $51.7 \%, \mathrm{P}<0.05)$. Three patients died due to multi-organ dysfunction syndrome.

Conclusions: Infectious complication is common in crush syndrome and faciotomy is a major factor in the prevalence of infection.

20

\section{Etiology, Renal Replacement Therapy and Prognosis in Old Patients with Acute Renal Failure}

Qiu Hong-yu', Liu Fang Zuo chuan ${ }^{* 1}$, Mi Xu-hua ${ }^{1}$, Fu Ping ${ }^{1}$, Huang Song-min ${ }^{1}$

Department of Nephrology, West China Hospital, SCU, Chengdu, PRC

Objective: The aim of this study was to elicit etiologic profiles of old patients with ARF and to determine the relationship between the methods of blood purification and prognosis.

Method: 288 patients with ARF admitted to our department were retrospectively investigated, the etiology, incidence of ARF 
clinical characteristics, the methods of blood purification and prognosis were analyzed.

Results: 101 patients with pre-renal ARF, the proportion of the elder patients with pre-renal ARF was higher than non-elder patients groups; loss of fluid from gastrointestinal tract was the main cause, especially in elder patients; 158 patients with renal ARF, there was higher proportion in the non-elder patients groups; drug associated ARF remains the highest proportion; antibiotic associated ARF remains the main cause, with the increasing trend about NSAIDs; 29 patients with post-renal ARF, the etiology was urinary tract stone, stenosis and tumors; There was no significant difference between the two groups in the days in hospital, the days of oliguria, the size of kidney, Serum urea nitrogen, creatinine; the proportion of the elder patients complicated with liver damage, MODS was higher; the mortality in elder patients is also higher; treatment of hemodialysis could reduce the mortality. Continuous renal replacement therapy couldn't significantly reduce the mortality compared with IHD.

Conclusions: Fluid loss of gastrointestinal tract and nephrotoxic drugs remains the primary cause in elder patients; the early use of HD could increase survival rate; the age ,the number of organ system injury were predictors of mortality; more attention should be paid to the elder patients, who often complicated with chronic diseases, more easily be attacked by ARF .

\section{1}

\section{Continuous Renal Replacement Treatment of the Renal Victims of Wenchuan Earthquake}

Qin Wei, Fu Ping, Mi Xuhua, Su Baihai, Tao Ye,
Chen Xiaolei, Li Jing

Division of Nephrology, Department of Medicine, West China Hospital, Sichuan University, Chengdu, Sichuan, China

Background: 1845 earthquake wounded patients were hospitalized in our Hospital after Wenchuan earthquake. 42 patients were treated with CRRT. Efficacy was reported in order to share our experiences.

Method: 42 patients were divided into 3 groups according to indications of CRRT. 24 patients complicated with MODS and sepsis were assigned into MODS group. 9 patients with MESS (mangled extremities severity score) score $>7$ were assigned into limb salvage group (LS). 9 patients with IHD uncontrolled volume overload or electrolytes disorders were assigned into hypermetabolism group (HM). All patients were treated with CVVH. Replacement fluid was given at a speed of 3-4L/h. Local citrate anticoagulation was applied.

Results: Significantly higher CK lever and APARCHE II score were noticed in LS and MODS group respectively (Table 1). Only 6 patients in MODS group died, which indicated a lower mortality comparing with Marmara earthquake. Dead patients were characterized as older; with severer injuries and higher APARCHE II score (Table 2). Extremities of 6 patients in LS groups were salvaged after treatment in stead of amputation. Volume overload and electrolytes disorders were corrected after a short period of CRRT treatment in all patients of HM group. APARCHE II score in each group was decreased significantly after CRRT treatment. Serum creatinine and BUN levels in each group was also decreased remarkably after treatment. Electrolytes disorders (hyperkalemia, hypocalcaemia and hyperphosphatemia) were controlled after treatment (Table 3). Dramatic reduction was also observed in serum CK, AST and Myoglobin (Myo) levels in each group. 7 months follow up indicated only 2 patients have developed into chronic renal failure. No severe complications were noticed during the CRRT treatment.

Conclusion: CRRT could efficiently treat patients with crush syndrome caused ARF.

\section{2 \\ Prospective Study of Spectrum of Acute Kidney Injury (AKI) in Sick, Hospitalized Children}

\author{
A. Sinha, P. Mehta, A. Bagga, P. Hari, R. Lodha \\ Department of Pediatrics, All India Institute of Medical \\ Sciences, Ansari Nagar, New Delhi, Delhi
}

Purpose: Although a definition for AKI has been proposed, there is little information regarding its incidence, etiology, predictors and outcome in children. We conducted this study to determine the incidence of AKI in hospitalized children, evaluate the progression between stages of AKI, and relate the stage and etiology of AKI to mortality.

Methods: We conducted a prospective observational study in all pediatric (1 month-18 yr) patients admitted to the intensive care unit (ICU) or through emergency services to the pediatric wards. Patients with known chronic kidney disease (CKD) stage 5, jaundice with total bilirubin $>5 \mathrm{mg} / \mathrm{dl}$ and hospital stay of $<24$-hr were excluded. AKI was defined and classified according to definitions proposed by the Acute Kidney Injury Network (Crit Care 2007, 11: R31). Standard indications were used for initiation of peritoneal dialysis (PD) or hemodialysis (HD).

Results: Patient characteristics are shown in Table 1. Of 526 admissions from February-September 2008, 59 (11\%) had AKI; of which 24 were in ICU (incidence in ICU 26\%). The maximum AKI stage seen was 1 in 15, 2 in 13 and 3 in 31 patients; progression of AKI stage was noted in 16 patients. The etiology included acute tubular necrosis (ATN) in $44(74.5 \%)$, rapidly progressive glomerulonephritis in $5(8.5 \%)$ and hemolytic uremic syndrome in $4(6.8 \%)$. Twelve (20.3\%) patients required PD and $9(15.3 \%)$ had HD. Recovery from AKI was complete (as defined as normal creatinine, urinalysis and blood pressure) in $22(37.3 \%)$ and partial (defined as abnormal creatinine or urinalysis, or presence of hypertension) in $18(30.5 \%)$ patients, while GFR $<15 \mathrm{ml} / \mathrm{min} / 1.73 \mathrm{~m}^{2}$ persisted in $19(32.2 \%)$

Table 1 Results

\begin{tabular}{|c|c|c|c|}
\hline & Total & AKI & Non-AKI \\
\hline Total number of admissions & 526 & $59(11.2 \%)$ & 467 \\
\hline Number admitted into ICU & 94 & $24(25.5 \%)$ & 70 \\
\hline $\begin{array}{l}\text { Age in months (standard } \\
\text { deviation, SD) }\end{array}$ & $49(31)$ & $70.3(45.9)$ & $46.3(22)$ \\
\hline $\begin{array}{l}\text { Duration of hospital stay in } \\
\text { days (standard deviation, SD) }\end{array}$ & 9.2.(6.3) & $12.9(9.7)$ & $8.7(5.6)$ \\
\hline
\end{tabular}


patients. Thirty (50.8\%) patients with AKI died while 7 (11.8\%) were discharged on RRT ( 3 on HD, 4 on ambulatory PD).

Presence of shock \& need for mechanical ventilation were significantly associated with risk of developing AKI, relative risks $(95 \%$ CI) being 3.7 (2.2-6.4) and 4.4 (2.7-7.2) respectively. AKI was associated with higher risk of dying [RR 5.7 (3.9-8.3)]; causes of mortality included septic shock (25), ARDS (5) and hemorrhage (4). In patients without AKI, sepsis was the chief cause of mortality.

Conclusions: AKI is common in hospitalized pediatric patients, with patients with shock, mechanical ventilation and those requiring ICU care being at increased risk. Patients with AKI had an increased mortality compared to those without AKI.

\section{Emerging Concepts}

23

\section{Building a Homogenous CRRT Team}

M.H. Chaaban, Y. Lee, O. Alfurayh

Department of Hemodialysis, King Fasal Specialist

Hospital and Research Center, Riyadh, Saudi Arabia

Historically, Continuous Renal Replacement Therapy (CRRT) is mainly a critical care procedure indicated for the treatment of acute renal failure patients who can not tolerate intermittent hemodialysis procedure and these patients are hemodynamically compromised with severe fluid overload, hypercatabolic or at risk of cerebral edema and are in intensive care units(ICU). CRRT remain primarily a nephrology treatment and it is considered as a slow hemodialysis procedure, thus the nephrology team involvement is required. In this presentation we will review the advantageous of multidisciplinary approach for a successful implementation of a CRRT Program.

Learning objectives for the abstract: At the end of this presentation the participant will be able to understand:

1. Illustrate how multidisciplinary collaboration can achieve optimal patients' care?

2. The consequence of role definition in creating healthy working environment, in a multidisciplinary patient care procedure.

\section{References:}

1 Daugirdas, J.T., Blake, P.G., Ing. T.S., Handbook of Dialysis: 44th Edition: 2007 Lippincott, Williams \& Wilkins, New York.

2 Molzahn, A., Butera, E., Contemporary Nephrology Nursing: Principles and Practice, 2nd Edition: 2006, ANNA, Anthony J., Jannetti, Inc., Pitman, New Jersey, pp $464-481$

3 Renal Replacement therapy for acute renal failure in children European guidelines 18.12.IPNA 2003 Valdemir strazdins etal.

4 Haig, K. et al., (2006) SBAR a Shared Mental Model for Improving Communication between Clinicians.

5 Martin, P et al. (2005) Satisfaction with Multidisciplinary Treatment team D\&T 34:1 P 12-19

\section{4}

\section{Early but Not Late Fluid Resuscitation was Associated with Improved Outcome in Septic Shock}

O.I. Udeozo, M.G. Selby, R. Cartin-Ceba, K.B. Kashani, E.N. Haugen, G. Ognjen

Department of Critical Care Medicine, Mayo Clinic, Rochester, MN, USA

Purpose: Adequate volume resuscitation is an important aspect of management in sepsis and in pre renal acute kidney injury. Recent studies lend support to aggressive resuscitation in early sepsis. However, exact volume or endpoints have not been defined. Here we attempt to show that continued volume infusion post initial resuscitation is not beneficial and may be associated with increased mortality.

Methods: From a prospective database of medical critically ill patients with septic shock we identified patients who developed acute kidney injury (AKI). Patients who denied research authorization and readmissions were excluded. Severity of illness, fluid resuscitation and fluid balance data at different time points after the onset of septic shock (the first 12 hours, 24-72 hours) were collected from the medical records and APACHE III database.

Results: Of the 390 patients who met the inclusion criteria median age (IQR, interquartile range) was 68(56-79), there were 180 (46\%) females, and 350 (89\%) Caucasians. BMI median (IQR) was 27 (23-33). Median Apache score was 87(67-105). 268 developed AKI and they were classified according to RIFLE criteria: Failure 122, Injury 86, Loss 1, and Risk 59.

Patients that survived to hospital discharge had a net median (IQR) fluid balance in the first 12 hours of $4625 \mathrm{~mL}$ (2429-6380) versus $3452 \mathrm{~mL}(1796-6193)$ in the patients that died ( $\mathrm{p} 0.025)$. In the period of 24 hours to 72 hours, patients that survived to hospital discharge had a net median (IQR) fluid balance of $4196 \mathrm{~mL}(348-24235)$ versus

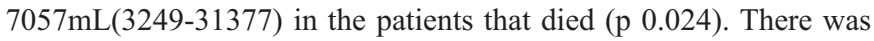
no difference in ICU length of stay between both groups of patients.

Conclusion: In accordance with current literature our study shows improved outcome with early volume resuscitation. However we were also able to show that continued volume infusion and positive fluid balance after 24 hours is not beneficial and may be harmful.

25

Risk Factors Associated with the Development of "End Stage" Renal Disease in ICU Survivors that Required Renal Replacement Therapy

R. Cartin-Ceba, O. Gajic, K. Banaei-Kashani

Department of Nephrology and Hypertension/Pulmonary and Critical Care, Mayo Clinic, Rochester, MN, USA

Purpose: The Risk, Injury, Failure, Loss and End stage (RIFLE) classification has been widely accepted for the definition of acute kidney injury (AKI); however, no study has described in detail the last stage of the classification: "End stage". We aim to describe the risk 
factors associated with the development of "End Stage" renal disease in ICU survivors that required renal replacement therapy (RRT).

Methods: Retrospective analysis of prospectively collected Acute Physiology and Chronic Health Assessment (APACHE III) database. Consecutive critically ill patients $>18$ years of age admitted to three ICUs of two tertiary care academic hospitals, from January 2003 through August 2006, excluding those who denied research authorization, chronic hemodialysis therapy, readmissions, and admissions for less than 12 hours for low risk monitoring. In order to compare the characteristics between the patients that required RRT but did not progress to End stage, we excluded from the comparison all patients that died before 3 months of RRT because they did not have the possibility to progress to "End stage" due to timing. Risk factors identified in the development of "End stage" were compared and analyzed in a multivariate analysis.

Results: 11644 patients were included in the study. The median age was 66 (interquartile range, 52-76), 90\% were Caucasians and $54 \%$ of the patients were male. Half of the patients developed AKI, and most of the patients were in the Risk and Injury stages. A total of 1065 (9.1\%) patients required renal replacement therapy (RRT), 415 (39\%) underwent continuous renal replacement therapy (CRRT) and 650 (61\%) underwent intermittent hemodialysis (IHD). After excluding 281 patients who died within 3 months of initiation of RRT, we found that 282 patients progressed to "End stage". After multivariable adjustment, the progression to "End stage" was associated with higher baseline creatinine, OR 1.19 per every increase in creatinine of $0.1 \mathrm{mg} / \mathrm{dL}(95 \% \mathrm{CI}, 1.11-1.29) \mathrm{p}<0.001$; and less frequent use of CRRT, OR 0.18 (95\% CI, 0.11-0.29) $\mathrm{p}<0.001$.

Conclusion: "End stage" renal disease after AKI requiring RRT in the ICU was more likely in patients with elevated baseline creatinine and those treated with intermittent hemodialysis.

\section{6}

\section{Epidemiology of Acute Renal Failure: Cross-sectional Hospital Based Study}

\author{
A. Kaul, R.K. Sharma, R. Tripathi, A. Gupta, N. Prasad, \\ S. Mandal \\ Department of Nephrology, Sanjay Gandhi Post Graduate \\ Institute of Medical Sciences, Lucknow, Uttar Pradesh, \\ India
}

It is crucial to know the incidence, etiology and clinical features of Acute renal failure, so as to promote prevention strategies and implement adequate resources for management of this treatment able etiology of renal failure. During a 5 yrs period a total of 240 patients cross sectionals were diagnosed with ARF, selected among 5499 patients admitted in nephrology accounting for 2.5 percentage of population.

Average age in the population was 38.8 yrs (range 15-65 yrs) with 9.1 percent population who were more than 60 yrs of age while 6.6 percent were less than 15 age. 58.9 percent were males while 99 patients were females out of 240 patients. 18.7 percent were biopsied while 197 were not. Aetiology of Acute renal failure (biopsied or not) was ATN (184), AGN (2), AIN(29) hus(8), cortical necrosis (10). Malarial ARF (10.4 percent), drug induced ATN (6.5 percent), snake bite leading to ARF 2.9 percent, obstrectical ARF 11.2 percent, heart disease precipitated ARF 6.9, while liver disease was etiology in 7.4 percent, Post operative ATN in 17.5 percent and 26.2 percent of ARF succumbed. 143 patients showing complete recovery and 14 percent showing partial recovery.

Rifle score for risk of ARF was 5 percent, injury in 11.6 percent while 88.3 percent were at risk to failure. 60 percent population was having sepsis with ARF with DIC in 41.2 percent population.

35.8 percent population required blood components (FFP, Platelets, blood) in view of DIC. Culture positivity was observed in 31.6 percent population, 44.1 percent presented in Hypotension at time of presentation. These were the patients who had sepsis with multi organ failure. Dialysis was indicated 83.3 percent population, 44.5 were given HD, 22.5 percent CRRT, 15 percent SLED and 2.5 percent underwent PD. Average Hospital stay of patients 10.8 percent (Less than 5 days), 47.5 percent ( 5 to 10 days), 38.7 required for more than 10 days duration.

Patient's outcome predictors having significant value were respiratory involvement (.009) ICU setting (.000), Hemoglobin less than $10 \mathrm{gm}$ percent ( .014$)$, Serum Albumin less than $3.5 \mathrm{gm}$ percent (.042). Heart disease (.01), Liver disease (.002) while renal outcome was predicted with significance was seen with less than 50 yrs age (.014), respiratory involvement (.000), Hypotension (.042), hemoglobin less than $10 \mathrm{gm}$ percent (.06), Serum Albumin less than 3.5 gm percent (.04), Culture positivity (.007), Heart disease (.000). Thus preventive strategies particularly in Peri and Post operative period, cardiac and chronic liver disease Population if handled with care, it can potentially decrease the incidence of ARF and Patients out come in at risk population.

27

\section{Outcomes Following Diagnosis of Acute Tubular Necrosis}

\section{L.S. Chawla', R.L. Amdur², S. Amodeo ${ }^{3}$, P.L. Kimmel', C.E. Palant}

${ }^{1}$ Department of Anesthesiology and Critical Care

Medicine, George Washington University Medical Center,

${ }^{2}$ Department of Psychiatry, Georgetown University School of Medicine, ${ }^{3}$ Research and Medical Service, Veterans Affairs Medical Center, ${ }^{4}$ Division of Renal Diseases and Hypertension, Department of Medicine, George Washington University Medical Center, Washington DC, USA

Background: After developing acute renal failure (ARF) or acute tubular necrosis (ATN), a proportion of survivors progress to chronic kidney disease (CKD). The long-term course of ATN is unknown because estimated glomerular filtration rate (eGFR), development of advanced CKD, and mortality in ARF and ATN and similar hospitalized patients with acute illness, without CKD, has not been assessed in contemporary care settings.

Methods: The U.S. Department of Veterans Affairs (VA) healthcare database was used to examine the renal function of 113,272 patients hospitalized either with ARF $(n=6694)$ or ATN $(n=441)$ over time. 75,137 patients hospitalized for acute myocardial infarction or pneumonia was designated controls. Outcomes of 31,000 patients with established CKD were assessed. Serum creatinine levels, eGFRs and mortality for up to five years after study entry were analyzed. 
Results: Renal function in survivors decreased over time in ARF, ATN, and control groups, but with significantly greater magnitude and rapidity in ARF and ATN. $15.6 \%$ of ARF and $21.8 \%$ of ATN patients developed Stage 4 CKD, as compared to $3.6 \%$ of controls ( $p$ $<0.01$ ). In Cox regression analysis adjusting for age, gender, and DM status, the diagnosis of ATN was associated with seven times the risk of progressing to CKD-4 (OR 7.1, SE 0.11, p <0.0001).

Conclusions: Patients with ARF or ATN had significant renal functional decline over time, and were at high risk for the development of advanced CKD. Survivors of ATN are high risk of death and development of CKD.

\section{8 \\ Clinical Study of 21 Pediatric Sepsis Cases Performed PMX-DHP}

H. Kitayama, N. Wada, T. Kawasaki, M. Yamada, A. Horie, M. Uehara

Department of Pediatric nephrology, Shizuoka Children's Hospital, Shizuoka, Japan

PMX (polymyxin B-immobilized fiber Column) can adsorb endotoxin. PMX-DHP was performed for sepsis patients. We used PMX-05R which produced by Toray company for 21 pediatric sepsis patients who have various back ground. Priming volume of PMX05R $(40 \mathrm{ml}$ for children) is less than PMX-20R(135ml, for adult).

Purpose: We studied efficacy of PMX-05R for various factors. The factors are age, body weight, diagnosis, PELOD score, mortality, kidney injury, other blood purification, blood pressure, $\mathrm{PaO} 2 / \mathrm{FiO} 2(\mathrm{PF}$ ratio), prognosis of renal function and neurological prognosis.

Result: Average age was 1 year and 11 months. Average body weight was $7.1 \mathrm{~kg}$. All patients were diagnosed Sepsis. Background diagnosis was congenital heart disease (ECMO 3 cases) (6 cases), intestinal disease (neonatal necrotizing enterocolitis, perforation of stomach) (5 cases), persistent pulmonary hypertension of newborn (3 cases), acute encephalopathy (4 cases), malignancy ( 2 cases), aspyxia at birth (2cases), congenital nephrotic syndrome (1case), inborn errors of metabolism(1 case), purulent meningitis (1case), VATER association(1 case) and neurological disorder(10cases)(duplication+). We safely performed CRRT for all cases without side effect.

Before performed PMX-05R, average PELOD score was 35.8 points. Estimated mortality was 0.80 due to PELOD. Practical mortality was 0.57 . In only one case there are no kidney injury. The others had kidney injury. Kinds of blood purification are CHDF(17cases), CHD(2cases), CHF(2cases), PEX(3cases) and PMX-DHP(21 cases).

We could check blood pressure of 18 cases in 21 cases. 13 cases blood pressure increased by $22.7 \mathrm{mmHg}$. 12 cases PF ratio could be checked. 9 cases PF ratio were under 200(ARDS). 2 cases PF ratio were from 200 to $300(\mathrm{ALI})$. 10 cases PF ratio increased to over 300 units.

Three months interval after performed PMX-DHP, 9 cases were examined creatinine clearance (Ccr). 4 cases renal function were normal range (average Ccr;119.4). 5 cases renal function decreased (average Ccr;63.7). Neurological state of 2 cases worsened than before sepsis.
Conclusion: From 1997 to 2001, survival rate was 9.1\% in our hospital without PMX-DHP. From 2002, in this study, survival rate achieved to $42.9 \%$ with PMX-DHP. PMX-DHP had good influence on blood pressure and PF ratio. We thought that these effects made survival rate better.

\section{9 \\ Neutrophil Gelatinase-Associated Lipocalin: (NGAL) Early Biomarker for Acute Kidney Injury (AKI) in the Setting of Cardiac Morbidity - A South Indian Experience}

\author{
P. Khanna', L.N. Kamaladevi², A. Mehrotra', G. Abraham², \\ P. Ambikapathi², M. Mathew ${ }^{2}$ \\ ${ }^{1}$ Sri Ramachandra University, Chennai, India, ${ }^{2}$ Madras \\ Medical Mission Hospital, Chennai, India
}

Aim: To study NGAL as an early biomarker for AKI in patients undergoing cardiac surgery with cardio-pulmonary bypass (CPB) and those with acute coronary event (ACE).

Methods: Prospective study on adults admitted to $\mathrm{CCU}$ in tertiary care centre, underwent CPB $(n=25)$ study A. Study B were patients with ACE $(n=18)$ admitted in CCU.

Pre-operative serum creatinine levels $\leq 1.2 \mathrm{mg} / \mathrm{dL}$ were included, those with pre-surgery/event creatinine $>1.2 \mathrm{mg} / \mathrm{dL}$ were excluded

Blood and Urine samples were collected pre-operatively and two hours post surgery, ACE were analyzed by ELISA method for urine NGAL, blood urea, creatinine, urine creatinine, urine sodium and urine specific gravity.

AKI was defined by rise in creatinine by $>50 \%$ compared to baseline. Diagnostic cut off for urinary NGAL was defined as above 250 $\mathrm{ng} / \mathrm{mL}$ for predicting AKI.

Descriptive analysis was done on Study A. Statistical analysis on Study B was performed using SPSS 11.0 with t-test and chi-square test. P-value $<0.05$ was considered significant.

Results: Study A ( $\mathrm{n}=25)$ had 19 males $(76 \%), 6$ females $(24 \%)$, $\mathrm{n}=8(32 \%)$ diabetics and $\mathrm{n}=7(28 \%)$ hypertensives. Mean age of the group was $45.28 \pm 14.48$ years. Mean NGAL was $40.55 \pm 24.88 \mathrm{ng} /$ $\mathrm{dL}$ pre-operatively and $40.79 \pm 61.28 \mathrm{ng} / \mathrm{dL}$ post operatively. Mean creatinine $0.89 \pm 0.19 \mathrm{mg} / \mathrm{dL}$ pre-operatively, $0.95 \pm 0.26 \mathrm{mg} / \mathrm{dL}$ post operatively. Mean serum urea $28.03 \pm 10.6 \mathrm{mg} / \mathrm{dL}$ pre-operatively, $26.4 \pm 8.1 \mathrm{mg} / \mathrm{dL}$ post operatively. Eleven patients had a slight rise in NGAL post-operatively and one patient had NGAL $124 \mathrm{ng} / \mathrm{dL}$ preoperatively to $326 \mathrm{ng} / \mathrm{dL}$ post-operatively, with normal serum creatinine levels but rise in serum urea levels on day three post operatively. None developed AKI.

Study B(n=18) had 17 males( 94.4\%), one female (5.6\%), 8 diabetic (44.4\%) and 7 hypertensive (38.9\%). Mean age was $65.18 \pm 12.69$ years. NGAL levels did not correlate significantly with creatinine after 24 hours $(\mathrm{p}=0.295)$, eGFR $(\mathrm{p}=0.283)$, urinary sodium excretion $(\mathrm{p}=0.635)$, blood urea level $(\mathrm{p}=0.455)$, urine creatinine $(\mathrm{p}=0.738)$ and urinary specific gravity $(\mathrm{p}=0.905)$. None developed AKI.

Conclusion: This prospective study guided us to institute appropriate measures to prevent AKI in cardiac patients who had comorbidities such as diabetes and hypertension. Rise in NGAL was below $250 \mathrm{ng} / \mathrm{dL}$ which was not predictive of AKI. 


\section{0 \\ Commercial Albumin Containing Caprylate as Conservative May be Limited to Exert Its Clinical Benefits as an i.v. Plasma Expander in Advanced Liver Failure with Renal Insufficiency}

\section{J. Stange, S. Mitzner, S. Strube, A. Goetze, J. Gruenert, J. Emmerich \\ Department of Internal Medicine, University of Rostock, Rostock, MV, Germany}

Background: The Standard of Care in the treatment of Complications of portal Hypertension in Liver Cirrhosis includes the infusion of Albumin. Specifically the therapy of refractory ascites, spontaneous bacterial peritonitis and hepatorenal syndrome require the use of albumin as a plasma expander, which is supported by prospective controlled trials. The advantage of albumin in comparison to synthetic plasma expanders (e.g. starch) has been associated with additional biological functions, as the ability to bind smaller molecules of pathophysiological relevance.

Unfortunately, commercial albumin must undergo the process of virus inactivation and storage (up to 5 years), both requiring the industrial addition of caprylate (=octanoate; OCT) and frequently addional N-Acetyltryptophan (NAT). Both substances bind to the benzodiazepine site of albumin and significantly reduce commercial albumins binding capacity for ligands, e.g. toxins.

The Ability to bind toxins relevant in Liver failure can be measured by testing the Albumin Binding Capacity (ABiC) for markers. Clinical studies have shown, that $\mathrm{ABiC}$ of Patients Albumin in Liver Failure is significantly reduced which correlates to the degree of liver disease (MELD and CTP, Klammt et al, EJGH 2007). This observation has been explained by the occupation of albumin by toxins which accumulate in liver failure.

Pharmaceutical Albumin Preparations are characterized by an extremely reduced Albumin Binding Capacity, a consequence of the (more than 5-10-fold per molecule) overload with OCT and NAT. If infused in patients with liver failure, those substances not only reduce the therapeutic effect of albumin by occupying the needed binding sites, but have also been shown to have undesired adverse events on hepatic encephalopathy, hemodynamic stability and renal function. As the liver plays an important role in the metabolism of OCT and NAT the risk to accumulate toxic levels by albumin infusion is even higher in liver disease. Regulatory Authorities have issued warnings about those adverse event risks.

Study Design: In order to identify the actual clinical consequences of OCT and NAT in albumin solutions applied in liver failure, a clinical protocol has been applied to compare the effect of albumin infusion with and without OCT and NAT in refractory ascites, spontaneous bacterial peritonitis and hepatorenal syndrome. 15 patients received standard albumin and 25 received albumin via a nanostructured charcoal filter (Hepalbin-Adsorbent, Albutec $\mathrm{GmbH}$, Rostock) designed to eliminate NAT and OCT. Plasma OCT, NAT, $\mathrm{ABiC}$ were detected and prognostic parameters (renal function and hepatic encephalopathy) were observed.

Results: The disturbed metabolism of OCT and NAT in liver failure resulted in a significant accumulation of both substances in the control group. None of the patients who received the albumin via the Hepalbin Adsorbent presented with an accumulation of OCT or NAT reaching toxic levels. $\mathrm{ABiC}$ remained unaffected by standard albumin infusion, whereas the infusion of albumin via the Hepalbin Adsorbent increased the patients $\mathrm{ABiC}$ significantly up to 24 hours, which could also explain the favourable clinical effects on hemodynamic, renal and cerebral function. The group receiving Albumin without OCT and NAT via the infusion filter showed less deterioration and more improvement in hepatic encephalopathy and renal function.

Conclusion: Although the clinical benefits of commercial albumin as a plasma expander in the described indications has been shown by controlled studies, its use has been challenged by many, specifically in light of its increasing price. The present data suggest that by applying albumin in advanced liver failure without exposing he patients to the potentially toxic effects of OCT and NAT albumins risk/benefit/ cost profile could be improved further and its use more convincing. Multicenter trials are in preparation to reproduce those observations.

\section{1 \\ Are Admission Plasma and Urine Values of Neutrophil Gelatinase Associated Lipocalin (NGAL) Associated with Mortality in Adult ICU Patients Requiring Continuous Renal Replacement Therapy (CRRT)?}

H.D.E. Geus', J.L.E. Noble1, F. Zij/stra'2, C. Ince', J. Bakker ${ }^{1}$

${ }^{1}$ Department of Intensive Care, ${ }^{2}$ Department of

Anesthesiology, Erasmus University Medical Center, Rotterdam, Zuid Holland, The Netherlands

Inroduction: We investigated the association of plasma and urine Neutrophil Gelatinase Associated Lipocalin (NGAL) values with mortality in patients requiring Continuous Renal Replacement Therapy (CRRT) due to Acute Kidney Injury (AKI III) in a heterogeneous adult ICU population.

Methods: A prospective cohort study was conducted, including 16 patients. After ICU admission plasma and urine samples were collected at 8 time points. NGAL measurements were performed using an Enzyme Linked Immunosorbent Assay (ELISA). All patients received continuous venovenous hemofiltration $(\mathrm{CVVH})$ in a standard 2 liter substitution post-dilution mode and citrate or heparin was used for filter anticoagulation. Admission plasma and urine NGAL values were compared between survivors and non-survivors using Mann-Whitney $U$ test for non parametric variables.

Results: Mean age $( \pm \mathrm{SD})$ in years, APACHE II ( \pm SD) scores and calculated creatinine clearance on admission $( \pm \mathrm{SD})(\mathrm{ml} / \mathrm{kg} / \mathrm{min})$ for surviving $(\mathrm{n}=7)$ and non surviving patients $(\mathrm{n}=9)$ requiring CRRT were comparable, $(60 \pm 15,23 \pm 6,34 \pm 21$ and $60 \pm 14,27 \pm 8,24 \pm 14)$. Between these groups the prescribed dialysis dose was $24 \pm 4$ vs. $28 \pm 8$ $\mathrm{ml} / \mathrm{kg} / \mathrm{h}$ respectively. The plasma NGAL value on admission $( \pm \mathrm{SEM})$ was significantly different between survivors and non-survivors 872 $( \pm 207)$ vs. $1785( \pm 320) \mathrm{ng} / \mathrm{ml}(\mathrm{p}=0.02)$ as was the urine NGAL value on admission $1784( \pm 482)$ vs. $3355( \pm 477) \mathrm{ng} / \mathrm{ml}$. $(\mathrm{p}=0.05)$

Conclusions: Admission plasma and urine NGAL values in adult ICU patients with AKI requiring CRRT are associated with survival. Our data indicate that plasma and urine NGAL values might be used to predict mortality in adult ICU patients with severe AKI requiring CRRT. 


\section{2 \\ Predictive Factors for Acute Renal Failure in Crush Injuries in Wenchuan Earthquake}

\author{
Ping Fu, Zhangxue Hu, Xiaoxi Zeng, Yuanmao Tu, \\ Jingyuan Liang, Ye Tao, Songmin Huang \\ Department of Nephrology, West China Hospital, Sichuan \\ University, Chengdu, Sichuan, China
}

Purpose of the Study: To investigate the clinical manifestations and predictive factors for acute renal failure (ARF) in crush injuries.

Methods: Medical records of 1751 victims of Wenchuan earthquake treated in West China Hospital were retrospectively reviewed. Crush injury patients were included for further analysis. Inclusion criteria: (1) Patients were injured due to prolonged, sustained compression on the body and manifested tense, edematous with compromised vascular circulation or neurological disturbances in the affected portion at admission; (2) patients had received amputation or incision decompression before admission. ARF was defined as: oliguria (Urine output $<400 \mathrm{ml} / 24 \mathrm{~h}$ ), Blood urea nitrogen $>40 \mathrm{mg} / \mathrm{dl}$, serum creatinine $>2 \mathrm{mg} / \mathrm{dl}$ or serum potassium $>6 \mathrm{mEq} / \mathrm{L}$ The clinical data of patients with crush injury, including demographic and injuries characteristics, surgical intervention before admission, general conditions on admission, complications and laboratory data, were extracted and analyzed.

Results: After Wenchuan Earthquake, 1751 patients were hospitalized in West China Hospital. 146 patients were diagnosed with crush injury, 34\% (50/146) with ARF caused by crush injuries. The ratio of patients aged between 15 and 20 years old in ARF group and non-ARF group was $42 \%$ and $12.5 \%$ respectively, while the average age did not differ significantly between the two groups. In univariate analysis, the extent of crushed body was larger in ARF group than non-ARF group. The duration of being compressed $>2$ hours, mean blood pressure (MAP), creatine kinase (CK) and aspartate transaminase (AST), as well as the rate of patients with systemic infections, medical complications or surgical interventions, were statistically higher in ARF group than in non-ARF patients, while higher albumin level and platelet count (PLT) were observed in non-ARF group. In multivariate analysis, the extent of crushed body, surgical intervention before admission, medical complications and CK showed independent association with ARF in crush injuries.

Conclusion: ARF is one of the common complications in crushed injury patients. Patients with severe crush injury and pathophysiologic conditions are more inclined to develop ARF. The extent and number of crushed parts of body, surgical intervention, medical complications and creatine kinase level are predictive factors for ARF in crush injuries.

\section{Technique Characteristics}

\section{Factors Influencing Dose Delivery in Continuous Venovenous Hemofiltration (CVVH)}

\author{
Z. Huang ', A. Attaluri', M. Khan' ', J. Letteri', W. Clark ${ }^{2}$ \\ ${ }^{1}$ Department of Mechanical Engineering, Widener \\ University, PA, USA, ${ }^{2}$ Gambro Intensive Care, PA, USA
}

Recent data from the ATN investigators (NEJM, 2008) and Tolwani (JASN, 2008) did not confirm the results of earlier studies (Ronco et al, Lancet 2000; Saudan et al, Kidney Int 2006) indicating a direct relationship between CRRT dose and patient survival. Compared to the earlier studies, the ATN and Tolwani studies differed in several ways, including a lower total dose $(\mathrm{mL} / \mathrm{kg} / \mathrm{hr})$, use of diffusion rather than convection, and/or use of pre-dilution (PRE) rather than post-dilution (POST). In this experimental study, the latter issue of dilution mode was explored, using POST CVVH (the exclusive mode in the Ronco study) as the reference. Clearance of small solutes (urea; creatinine) and middle molecules (vancomycin; inulin) was measured in POST and varying degrees of mixed (PRE/POST) dilution, using the Prismaflex system and a $1.4 \mathrm{~m} 2$ filter (Gambro). The different PRE conditions were $0 \%$ (pure POST), $25 \%, 50 \%, 75 \%$, and $100 \%$ (pure PRE) with a constant blood flow rate $(290 \mathrm{~mL} / \mathrm{min})$ and replacement fluid $(\mathrm{RF})$ rate $(3 \mathrm{~L} / \mathrm{hr})$. The results $(\mathrm{mL} / \mathrm{min} ; \mathrm{N}=3$ for each condition) are shown in the table.

For a given RF rate, the balance between PRE and POST administration of RF has a predictable effect on small solute clearance. However, PRE/POST variation does not have a significant impact on middle molecule clearance. Although the specific solute class upon which effluent-based CRRT dosing is based has not been determined, these data should be considered in the interpretation of recent dose/ outcome trials.

Table for abstract 33

\begin{tabular}{lllll}
\hline \% Pre-Dilution & Urea & Creatinine & Vancomycin & Insulin \\
\hline 100 & $35.1 \pm 0.7$ & $35.5 \pm 0.3$ & $36.7 \pm 3.8$ & $33.6 \pm 3.1$ \\
75 & $41.1 \pm 0.4$ & $40.9 \pm 0.6$ & $32.1 \pm 0.8$ & $32.1 \pm 1.7$ \\
50 & $45.0 \pm 0.7$ & $45.3 \pm 0.4$ & $32.7 \pm 2.3$ & $33.8 \pm 0.9$ \\
25 & $51.5 \pm 1.3$ & $50.6 \pm 0.9$ & $35.2 \pm 1.5$ & $35.5 \pm 1.1$ \\
0 (POST) & $54.0 \pm 1.2$ & $54.0 \pm 1.2$ & $31.9 \pm 0.6$ & $34.7 \pm 5.6$ \\
P value (ANOVA) & $\mathrm{P}<0.0000001$ & $\mathrm{P}<0.00000001$ & $\mathrm{P}=0.074$ & $\mathrm{P}=0.71$ \\
\hline
\end{tabular}




\section{4 \\ Characterization of Blood Circuit Pressures in Pre-Dilution Continuous Veno-Venous Hemofiltration (CVVH) in Severe Renal Failure}

C.-S. Tan, H.-K. Tan, H.-L. Choong, C.-M. Chan

Department of Renal Medicine, Singapore General

Hospital, Singapore

Introduction: Circuit pressure changes in pre-dilution continuous veno-venous hemofiltration $(\mathrm{CVVH})$ are not well characterized. Analysis of the circuit pressure data may be useful in facilitating pre-emptive circuit changes, thereby avoiding unplanned treatment downtimes. Method: Single centre, retrospective study of 13 critically ill intensive care unit (ICU) patients with severe renal failure requiring dialysis. All cases were treated with heparin free CVVH. Pre-dilution CVVH was performed using Hemosol HF replacement fluid (Gambro, Lund, Sweden). Circuits were categorized into "clotters [C]" or "non-clotters [NC]" depending on whether they clotted $<24$ hours or $\geq 24$ hours from the start of CVVH, respectively. Four real-time circuit pressure readings (in $\mathrm{mmHg}$ ) were obtained from the Prisma CRRT machine (Hospital, Lyon, France).

Results: A total of $63 \mathrm{CVVH}$ circuits $(\mathrm{C}: \mathrm{NC}=19: 44)$ were analyzed and all of them clotted spontaneously eventually. Circuit lifespan $\mathrm{C}$ vs. $\mathrm{NC}$ was: $10.6 \pm 6.6$ vs. $43.6 \pm 19.0$ hours, $\mathrm{p}<0.05$. The baseline return pressure (RP), filter pressure (FP), access pressure (AP) and effluent pressure (EP) between $\mathrm{C}$ and $\mathrm{NC}$ were not statistically significant. Comparing $\mathrm{C}$ vs NC, 6 hours before clotting- EP6: $29 \pm 36$ vs. $-14 \pm 46, p=0.006 ; 4$ hours before clotting, EP4: $22 \pm 45$ vs. $-15 \pm 54, \mathrm{p}=0.024$; and, at 2 hours before clotting, EP2: $6 \pm 50$ vs. $-50 \pm 73, \mathrm{p}=0.013$. Filter pressure (FP) $\mathrm{C}$ vs. NC 6 hours before clotting- FP6: $198 \pm 27$ vs. $213 \pm 44$, p=0.204; 4 hours before clotting, FP4: $193 \pm 47$ vs. $223 \pm 38, p=0.015$; and, at 2 hours before clotting, FP2: $192 \pm 47$ vs. $226 \pm 44, p=0.009$. The RP and AP in C vs NC at 6,4 and 2 hours before clotting were not statistically significant.

Conclusions: Access and return pressures were comparable in both "Clotters" and "Non-clotters". However, non-clotting circuits had significantly worse filter pressures and effluent pressures, starting from 4 hours and 6 hours before terminal clotting, respectively. Adverse filter and effluent pressures do not appear to affect filter longevity in pre-dilution CVVH circuits primed with dilute heparin saline. Real time monitoring of pressure readings during CVVH do not reliably predict filter longevity.
35

Online Hemodiafiltration (OL-HDF), a "New" Renal Replacement Therapy for ICU-Acute Renal Failure (ARF): Evaluation of its Tolerance, Efficiency and Infectious Risk

L. Amigues ${ }^{1}$, J.P. Delabre1, H. Ramet ${ }^{2}$, A. Jaussent ${ }^{3}$, M.C. Picot ${ }^{3}$, J.P. Cristo/2

${ }^{1} \mathrm{CHU}$ Lapeyronie, Medical Intensive Care Unit, Herault, France, ${ }^{2}$ Department of Biochimie, CHU Lapeyronie, Montpellier, Herault, France, ${ }^{3}$ Department of Informatic and statistic, CHU Lapeyronie, Herault, France

Though OL-HDF is the gold standard therapy for chronic dialysis patients, it is however not yet widespread used in critically ill patients. This study was underwent to evaluate the efficiency and the tolerance of OL-HDF and to appreciate infectious and inflammatory consequences of its use in ICU-ARF patients.

Methods: 30 patients $(59.7 \pm 14.7$ years old, SAPS II $=58.9 \pm$ 20.5) were included for 72 HDF session's analysis (blood flow rate $300 \mathrm{ml} / \mathrm{mn}$, dialysate $500 \mathrm{ml} / \mathrm{mn}$, predilution infusate $100 \mathrm{ml} / \mathrm{mn}$, time of session $227.6 \pm 24.74 \mathrm{mn}$ ). Tolerance of OL-HDF sessions was estimated by: hypotension event $(20 \%$ reduction of mean arterial blood pressure), increase in vasopressors needs and pyrogenic reaction $\left(>1^{\circ} \mathrm{C}\right)$. Quality of dialysis was appreciated with $\mathrm{Kt} / \mathrm{V}$, reduction rates $\left(\mathrm{RR}=[1-(\mathrm{Cpost} / \mathrm{Cpre})]^{*} 100\right.$ where Cpre and Cpost are respectively solute concentration measured before and after each session) and instantaneous whole blood clearance $(\mathrm{K})$ for urea, creatinin and beta-2 microglobuline measured after $60 \mathrm{mn}$ and net ultrafiltration interuption $(\mathrm{K}=\mathrm{Qb} *($ Cart - Cven)/Cart) were $\mathrm{Qb}$ is effective blood flow, Cart and Cven are solute concentration in arterial and venous blood line). The infectious risk was investigated by weekly bacteriological and endotoxinic analysis of dialysate and infusate (Fresenius 5008 OL generators) and of samples from 3 different points of the ultrapure water line production (Fresenius AQ 1250). An eventual proinflammatory effect was appreciated before and after each OL-HDF sessions by systemic super oxyde anion production as measured in the venous blood by chemoluminescence technique before and after PMA stimulation.

Results: 1 . Tolerance: hypotension: 13 sessions (18.1\%), increase vasopressors : 2 sessions, pyrogenic reaction: 1 session. For the 72 sessions, mean MAP: $87.9 \pm 16.3 \mathrm{mmHg}$ before, $90.8 \pm 16.8 \mathrm{mmHg}$ after. 2. Efficiency: $\mathrm{Kt} / \mathrm{V}=1.13 \pm 0.27$, reduction rates and instantaneous clearance was respectively $62.3 \pm 8.7 \%$ and $235.1 \pm 22.9 \mathrm{ml} / \mathrm{mn}$ for urea, $54.2 \pm 6.6 \%$ and $194.2 \pm 23.4 \mathrm{ml} / \mathrm{mn}$ for créatinine and $49.9 \pm$ $9.3 \%$ and $81.4 \pm 29.4 \mathrm{ml} / \mathrm{mn}$ for beta- 2 microglobuline. 3 .Infectious risk: 798 infusate, dialysate or water line samples were analyzed: 3 bacteriologicals results were non conformable ( 2 dialysates, 1 infusate from different generators). Superoxide anion production before and after sessions was stable: $0.15 \pm 0.09 \mathrm{bit} /$ leucocyte versus $0.14 \pm 0.11$ bit/leucocytes before stimulation and $0.47 \pm 0.34$ bit/leucocyte versus $0.54 \pm 0.40 \mathrm{bit} / \mathrm{leucocyte}$ after PMA stimulation.

Conclusion: OL-HDF in acute renal failure patients is effective and well tolerated. Its use in ICU doesn't add any risk for patients but need to be regularly controlled by endotoxinic and bacteriological survey. 
36

\section{Continuous Renal Replacement Therapy Using Dialysate and Replacement Solutions Containing $140 \mathrm{mEq} / \mathrm{L}$ of Sodium is Associated with the Development of Hyponatremia}

\section{E.G. Clark, T. Podymow}

Division of Nephrology, McGill University Health Center, Montreal, OC, Canada

Purpose: One of the goals of continuous renal replacement therapy (CRRT) is to maintain normal electrolyte balance. CRRT dialysate and replacement solutions containing a sodium concentration of $140 \mathrm{mEq} / \mathrm{L}$ are routinely used. Despite this, the development of hyponatremia in patients on CRRT has been observed but is not well described. We sought to characterize the changes in serum electrolyte values that occur following the initiation of CRRT using a standard commercially available solution with a sodium concentration of $140 \mathrm{mEq} / \mathrm{L}$.

Methods: A retrospective analysis was conducted on fifty consecutive patients who received CRRT at our center between November 2007 and September 2008. Demographic data, admission diagnoses, severity of illness scores and CRRT characteristics were recorded. In addition, serum electrolyte, creatinine and urea levels were recorded prior to the initiation of CRRT and at consecutive 24-hour intervals while patients remained on CRRT, for up to five days.

Results: Thirty-six patients who received CRRT for over 24-hours were included in the study. The mean age of patients was 56 years with a mean APACHE II score of 31. Twenty-two patients received continuous venovenous hemodiafiltration with all but two of the remaining patients receiving continuous venovenous hemofiltration. The mean serum sodium level prior to initiation of therapy was $136 \mathrm{mEq} / \mathrm{L}$. After 24, 48, 72, 96 and 120 hours of CRRT, mean serum sodium levels were 134, 132, 132, 131 and $132 \mathrm{mEq} / \mathrm{L}$ respectively. Using a paired t-test, the differences from baseline were found to be statistically significant beginning at 24-hours following initiation of CRRT ( $p<0.01$ for all values). Notably, after 48 hours of CRRT, only two patients had recorded serum sodium values over $134 \mathrm{mEq} / \mathrm{L}$.

Conclusion: The initiation of CRRT at our center is associated with the subsequent development of hyponatremia. One possible explanation relates to the use of replacement and dialysate solutions that contain $140 \mathrm{mEq} / \mathrm{L}$ of sodium. Although the clinical implications of this finding are unclear, it may be practical to consider increasing the sodium concentration in replacement and dialysate solutions for those patients at high risk of complications associated with hyponatremia or hypo-osmolality.

\section{7 \\ Hemofilter Clotting in CRRT: Evaluating Factors and New Techniques to Improve Hemofilter Survival}

K.S. Sachdeva, A.S. Podoll, K.W. Finkel, T. Dang, V. Gupta

Department of Renal Diseases and Hypertension, University of Texas school of public health, Houston, TX USA

Objective: Identify variables that effect hemofilter life in patients undergoing Continuous renal replacement therapy (CRRT) in order to investigate new strategies to improve hemofilter survival and delivered dialysis dose.

Design: A prospective analysis of filter survival in 32 patients undergoing CRRT. The mean age of the population was 57 years of age and was composed of 20 men and 12 women of which $53 \%$ were Caucasian, 35\% were African-American and $12 \%$ were Hispanic. The reasons for initiating CRRT were ATN (20), sepsis (8), ESRD (3) and cardiorenal syndrome (1). $71.9 \%$ of patients required pressors, $96.9 \%$ were mechanically ventilated and $93.8 \%$ were oliguric. The average SOFA score was 10.7 . All patients were initially started on CRRT without any form of anticoagulation. After premature hemofilter clotting within the first 24 hours, ten patients (31\%) received regional citrate anticoagulation (RCA). Overall, 21 patients died and four regained renal function. Of the eight patients discharged on dialysis, three ultimately recovered renal function.

Results: Mean hemofilter life was 26.9 hours. The mean number of hemofilters per patient was 4.8 and the mean number of days on CRRT was 6.7. In patients whose hemofilter clotted within first 24 hours of initiation of CRRT, those who received RCA had a mean filter life of 24.5 hours while those who did not had a mean filter life of 12.9 hours. Of the ten patients on citrate, one developed metabolic alkalosis and 4 had hypocalcaemia. Multivariant analysis found no association with SOFA score, amount of administered blood products, total parenteral nutrition, dose of vasopressors, type of ICU or nursing-to-patient ratio. A significant association of hemofilter failure was found with femoral dialysis catheters and patient BMI $>35$.

Conclusion: In critically ill patients treated with CRRT, frequent hemofilter clotting increases blood loss, costs, and results in inadequate dosing of dialysis. As previously demonstrated using RCA improves filter life but has potential complications. Likewise, systemic heparin anticoagulation is associated with bleeding and HIT. Therefore, we are undertaking a randomized controlled trial to compare RCA, increased blood flow, and convective versus diffusion based therapy in patients who have frequent hemofilter failure.

38

\section{Concurrent ECMO, CRRT and TPE}

T.A. Mottes, S.W. Walters, D.B. Kershaw, G.M. Annich

University of Michigan, Ann Arbor, MI, USA

Continuous Renal Replacement (CRRT) has been successfully used with Extracorporeal Membrane Oxygenation (ECMO), usually with an inline filter requiring separate infusion pumps for dialysate 
and ultrafiltration management. There are limitations with dialysis prescribed and delivered dose in this system. Additionally, Total Plasma Exchange (TPE) treatments have been reported in concurrent with ECMO. With the indication for Total Plasma Exchange (TPE) increasing and the standards for dialysis prescriptions changing, we report a case of a 12 years-old diagnosed with necrotizing pneumonia as result of blastomycoses that presented in Acute Respiratory Distress Syndrome requiring ECMO. CRRT was initiated for acute renal failure using the standard setup, with a high dialysis dose required for adequate clearance. The limitation on external pump rates with the current system prevented us from achieving appropriate dialysis. As a result, the Prismaflex ${ }^{\circledR}$ (HF 1000) was added inline to the ECMO circuit, easily delivering our prescribed dialysis dose. The CRRT access line was connected post-blood pump on the ECMO circuit and the CRRT return line was connected pre-blood pump into the bladder of the ECMO circuit. The Prismaflex ${ }^{\circledR}$, with the capability for the positive access pressures, allowed us to provide adequate Blood Flow (250 ml/min), Dialysate Flow (3000 mL/hour) and Replacement Fluid (3000 mL/hour) Rates. Additionally, the patient required TPE. For this, an additional extracorporeal blood circuit using the Prisma ${ }^{\circledR}$ (TPE 2000) was also placed inline with the ECMO-Prismaflex ${ }^{\circledR}$ circuit. The TPE-Prisma ${ }^{\circledR}$ access line was connected pre-blood pump on the venous side of the ECMO circuit and the TPE-Prisma ${ }^{\circledR}$ return line was connected to the Prismaflex ${ }^{\circledR}$ return line in the ECMO circuit bladder. ECMO with CRRT and a total of 3 TPE treatments were performed simultaneously, without complications. Of note, only standard ECMO anticoagulation was required. This approach is particularly useful in complicated ECMO patients when multiple extracorporeal therapies are necessary.

\section{9 \\ Contamination of Commercial Albumin with Octanoate is a Limiting Factor for the Efficacy of Albumin Dialysis in Liver Support}

\section{J. Stange, S. Strube, S. Koball, S. Klammt, H. Hickstein, J. Hentschel \\ Department of Internal Medicine, University of Rostock, Rostock, MV, Germany}

Background: Commercial albumin is highly contaminated with octanoate and n-acetyltryptophane (NAT) which participate in the pathogenesis of hepatic coma and hemodynamic instability in liver failure. Enrichment of dialysate with albumin (Single Pass albumin dialysis; SPAD) has been proposed as a method of liver support in liver failure, however, recent reports have shown that stabilizer contamination is associated with octanoate and $\mathrm{N}$-acetyltryptophane intoxication. The MARS recycles the albumin dialysate by sorbents and dialysis, hence uses less albumin.

Aim: To compare the amount of octanoate intoxicating the patients during SPAD versus MARS treatment and to investigate the effect of octanoate on dialysate albumins ' binding characteristics.

Method: SPAD was carried out according to Sauer et al. MARS was applied using a comparable blood flow $(150 \mathrm{ml} / \mathrm{min})$ and using $100 \mathrm{~g}$ albumin in the recycling circuit, the flow rate was adjusted to $150 \mathrm{ml} / \mathrm{min}$. Three patients (one female, two males) on liver support therapy were crossed over from SPAD versus ECAD and back again.
Octanoate and NAT was detected by GC or HPLC respectively before and after dialyzer and in the dialysate solution. Total Octanoate entry was calculated. Before starting the treatment, the benzodiazepine binding site of dialysate albumin was characterized by its capacity to bind the binding site marker dansylsarcosine.

Results: During 8 hours of SPAD a total of 36,5 millimol octanoate was transferred from the dialysate into the patients blood. In MARS, the detectable octanoate concentration in the albumin dialysate after the preparation and immediately before patient connection was $3.6+/-0.46 \mathrm{mmol} / \mathrm{l}$. During 6 hours of MARS, a total of 5,2 millimol octanoate was transferred from the dialysate into the patients blood.

The binding capacity of dialysate albumin for dansylsarcosine before initiation of the treatment in SPAD was $50 \%$ only, compared to that of the control $(100 \%)$. Although the octanoate load in the MARS albumin circuit was lowered during the preparation to $1.6 \mathrm{~mol}$ per $1 \mathrm{~mol}$ albumin, the binding capacity of dialysate albumin in MARS for the benzodiazepine marker dansylsarcosine was still significantly lower $(65 \%)$ than the binding capacity of healthy control albumin.

Conclusion: Although MARS is associated with significantly less octanoate intoxication than SPAD, there is still an octanoate/ albumin ratio higher than 1 in MARS albumin dialysate, which is associated with a severely reduced binding capacity at the benzodiazepine binding site. A stabilizer free albumin as dialysate component is discussed as an option to improve the efficacy and safety of albumin dialysis.

\section{Use of Needleless Port on CRRT Circuit to Minimize Risk of Needle Stick Injury

\author{
C.A. Hansen, N.G McAfee, J. Symons \\ Department of Dialysis, Seattle Childrens, Seattle, WA, \\ USA
}

Purpose: Needle stick injuries can lead to serious or fatal infections. Health care workers who use needles are at increased risk of needle stick injury. Our CRRT device required the use of a syringe and needle or a sharp plastic connector for blood draws to monitor activated clotting times or ionized calcium. To reduce the risk of needle stick injury we sought a method to use a 3-way stopcock for drawing blood without the use of needles.

Methods: We initially tried a regular 3-way stopcock with a needleless cap on it placed in the blood line. This leaked, resulting in more potential exposure to blood. We requested a custom 3-way stopcock with a MicroCLAVE® device (ICU Medical, Inc., San Clemente, CA) permanently affixed. This device had no cap that could leak, very low dead space, and neutral displacement, which we felt would reduce any impact on blood flow. We placed this [TELL US EXACTLY WHERE].

Results: Staff draw blood easily without needles. There has been no leakage. We have not seen flow restrictions with this device in place.

Conclusions: This current 3-way stopcock has been very successful. We find this a very reliable method of obtaining blood from the circuit without the use of needles. 


\section{1 \\ Comparison of Tego Connectors and Normal Saline with Heparin Locking in Tunneled Catheters}

\author{
A.K. Kamra, S. George, D. Wadehra, N.K. Wadhwa \\ Division of Nephrology, State University of New York at \\ Stony Brook, NY, USA
}

In hemodialysis (HD) patients with catheters as vascular access, heparin is usually used as a locking agent to maintain catheter patency. Potential risks associated with heparin use such as heparin induced thrombocytopenia and bleeding preclude the use of heparin in these patients. Since 2005, the TEGO connector has been commercially available for HD catheters. The TEGO is designed as a neutral displacement connector, so when the blood tubing or a syringe is removed from the TEGO, there is no reflux of blood into the catheter lumen, thus eliminating the need for a 'Heparin Lock'. All patients with tunneled HD catheters received TEGO connectors with saline locking for acute HD at our institution since September 2008. We collected data on these patients from September 2008 to December 2008. This data was compared to a historical group that used heparin as a locking agent without TEGO connectors from March 2008 to August 2008. Heparin was used for 211 dialysis treatments in 14 patients ( 9 male and 5 female, mean age-62.5 years). The TEGO connector was used for 126 dialysis treatments in 15 patients (10 male and 5 female, mean age-66.6 years). The TEGO connector was connected to the catheter and blood tubing at the start of HD on day one and kept in place for the span of three dialysis treatments. The data was collected on catheter maneuvering, alteplase use, and catheter dysfunction. Maneuvering was defined as the reversal of lines or any change in patient position to obtain prescribed blood flow rate. Alteplase was used in patients with venous pressure $>250 \mathrm{~mm} \mathrm{Hg}$, blood flow delivered $<200 \mathrm{ml} / \mathrm{min}$ or Arterial Pressure $>-250 \mathrm{~mm}$ $\mathrm{Hg}$. Catheter dysfunction was defined as blood flow rate $<300 \mathrm{ml} /$ $\mathrm{min}$, arterial pressure $<-250 \mathrm{~mm} \mathrm{Hg}$ or venous pressure $>250 \mathrm{~mm} \mathrm{Hg}$, inability to aspirate blood freely and frequent pressure alarms. Data is summarized in the table below:

Catheter maneuvering, alteplase use and catheter dysfunction were similar in both groups. Preliminary data suggests that TEGO connectors may be an alternate choice to heparin in patients undergoing $\mathrm{HD}$ in the acute setting.

\begin{tabular}{llll}
\hline & Heparin $(\mathrm{n}=211)$ & $\begin{array}{l}\text { Tego with saline } \\
(\mathrm{n}-126)\end{array}$ & p value \\
\hline Maneuvering & $35(17 \%)$ & $30(24 \%)$ & 0.1 \\
Alteplase & $17(8 \%)$ & $7(6 \%)$ & 0.38 \\
Dysfunction & $21(10 \%)$ & $21(17 \%)$ & 0.07 \\
\hline
\end{tabular}

\section{2}

\section{Estimating Glucose Caloric Impact of CRRT Utilizing Regional Citrate Anticoagulation with ACD-A}

\author{
J. Macnowski, B.A. Mueller, A.M. Vilay, M. Heung \\ University of Michigan, Ann Arbor, MI, USA
}

Background: Regional citrate anticoagulation is increasingly used to maintain circuit patency in CRRT. ACD-A is a commonly used citrate formulation which contains $2.45 \%$ dextrose and may be a significant source of unrecognized caloric intake in critically ill patients.

Purpose: To develop a model to project glucose clearance and delivered glucose caloric load for patients receiving CRRT using ACD-A based anticoagulation.

Methods: Utilizing mass-transfer characteristics of glucose and other low molecular solutes, we developed a computer model of glucose clearance and delivery during dialysis. Independent variables in this model include the following prescription factors: mode of CRRT (CVVH, CVVHD, CVVHDF), blood flow rate (BFR), pre- or postfilter replacement fluid inputs, ACD-A flow rate, dialysate flow rates (DFR), ultrafiltration rate, and glucose concentrations of the input fluids. The model was applied to a range of prescriptions commonly encountered at our institution and to actual prescriptions of patients receiving CRRT.

Results: In the model, all standard initial CRRT prescriptions at our institution resulted in net positive glucose caloric delivery. Increasing ACD-A rate was the factor most significantly associated with increased caloric load delivery. Increasing BFR and decreasing plasma glucose concentrations also resulted in an increased caloric load, albeit to a lesser degree than ACD-A rate. Conversely, increasing DFR was strongly associated with a fall in delivered glucose calories. When applied to 12 consecutive patients receiving CVVHD in our institution, the CRRT-delivered caloric load ranged from 30 to $328 \mathrm{kCal}$ per day (mean $183 \mathrm{kCal}+86 \mathrm{kCal}$ ). Compared to estimated daily caloric needs, on average this represented an additional $10 \%$ in calories (range $1.9-21 \%$, SD $+5.0 \%$ ).

Discussion: Hypercaloric feeding, unbalanced caloric composition and hyperglycemia are associated with adverse clinical outcomes in critically ill patients. Our model suggests that standard CRRT prescriptions using ACD-A anticoagulation can result in excess glucose calorie delivery, which can result in overfeeding if unrecognized. This model was applied to the CRRT protocol at our institution, but is applicable to any CRRT protocol involving ACD-A.

Conclusion: In our model, the use of ACD-A in CRRT is associated with a significant and variable glucose caloric load for patients. Further in vitro and in vivo studies are planned to verify these results. Application of this model may provide real-time bedside guidance in the care of critically ill patients. 


\section{3 \\ Use of Anticoagulant Citrate Dextrose (ACD) as Regional Anticoagulation in CRRT is Effective, Improves Filter Life and Decreases Treatment Cost}

\section{G. Shidham, B. Hixon-Vermillion, K. Mount, J. Parrish \\ Department of Nephrology, The Ohio State University Medical Center, Columbus, OH, USA}

Purpose: Anticoagulation is an important component of continuous renal replacement therapy (CRRT). Systemic anticoagulation carries risk of bleeding, making regional anticoagulation a safe alternative.

Methods Used: The ACD protocol was implemented in June 2007 across all intensive care units at Ohio State University Medical Center. Retrospective data was collected regarding filter life on patients who were initially not on any anticoagulation and were later started on ACD for regional anticoagulation. Titration of ACD was based on CRRT post-filter ionized calcium. We compared filter life with and without citrate anticoagulation in the same patient, acting as self control.

Summary: Data from 16 patients was collected. Average length of therapy without anticoagulation was 2.73 days and mean length of ACD anticoagulation was 3.27 days. The post-filter ionized calcium goal was achieved in $90 \%$ of patients at 1 hour on ACD. Average filter life without anticoagulation was 12.63 hours, compared to 33.78 hours with ACD. Of all the patients receiving ACD, $39.5 \%$ of filters lasted for 72 hours, being changed at that time based on hospital policy.

While receiving no anticoagulation, length of therapy was 43.76 days using a total of 71 filters, compared to 52.31 therapy days using 38 filters. Cost to the patient for filter and filter changes while on no anticoagulation was $\$ 2,420.74$ /day compared to $\$ 1,083.84$ while on ACD.

Conclusion: Regional anticoagulation using ACD improved filter life and decreased the cost of treatment to patients receiving continuous renal replacement therapy.

\begin{tabular}{lll}
\hline & 16 patients & 16 patients \\
\hline Anticoagulation & No Anticoagulation & ACD \\
Average therapy (days) & 2.73 & 3.27 \\
Average filter life (hours) & 12.63 & 33.78 \\
Total \# filter used & 71 & 38 \\
Cost to patient for filter \& & $\$ 2420.74$ & $\$ 1083.84$ \\
filter change/day & & \\
\hline
\end{tabular}

14th International Conference on Continuous Renal Replacement Therapies (CRRT)
44

\section{A Systematic Approach to Improving Safety in CRRT Delivery}

\author{
M. Heung ${ }^{1}$, A. Kakulavaram², J. Jamieson ${ }^{1}$ \\ ${ }^{1}$ Department of Internal Medicine/Nephrology, University \\ of Michigan, Ann Arbor, MI, USA, ${ }^{2}$ University of Rochester
}

Purpose: To characterize incidence, risk factors and consequences of error-related adverse events during CRRT delivery. To implement systematic interventions in order to reduce the risk of future errors.

Methods: We conducted a single center review of incident reports filed from intensive care units during the 4 year period of 2004 thru 2007 to identify errors related to CRRT delivery. Identified cases were subject to root cause analyses to determine underlying contributing factors. Additionally, random chart audits of patients receiving CRRT were performed to evaluate the incidence of unreported errors. Clinical characteristics and outcomes of patients suffering adverse events were examined.

Results: During the study period there were 2216 intensive care unit incident reports filed and $39(1.75 \%)$ were identified as being related to CRRT. Ten incidents were excluded as being related to equipment failure and without adverse patient consequences, leaving 29 incidents. Over the same period our institution performed a total of 8124 CRRT treatment-days, giving an error-related adverse event rate of 1 in 280 CRRT patient-days ( $0.36 \%)$. Most (55\%) events were related to use of regional citrate anticoagulation. Two events leading to excess citrate administration resulted in patient cardiac arrest, although no incident resulted directly in patient death. The hospital mortality rate of patients suffering an error-related adverse event on CRRT was $46 \%$ (13/28). In non-survivors, the mean number of days between the reported error and death was 13.2 (range 0 to 31). Chart audits did reveal additional unreported errors, primarily in failure to properly follow protocols; no such errors appeared to result in significant adverse clinical consequences.

Discussion: Based on our analysis, several high risk areas were identified and changes implemented. Interventions include an audit of delivery/storage of CRRT solutions and the introduction of a dedicated calcium infusion bag with visual and tactile cues, both aimed at reducing the risk of incorrect fluid substitution. Additionally, we implemented a program to standardize nursing training and competency for CRRT delivery with a particular focus on error reduction.

Conclusion: CRRT can be delivered safely with a low incidence of medical errors utilizing well-established standardized protocols and experienced personnel. Our review of CRRT-related medical errors identified high risk areas which subsequently led to interventions aimed at minimizing these errors. We encourage other institutions to conduct and share similar studies to promote overall safer CRRT practices. 
Targeted Interventions

\section{5 \\ Continuous Veno-Venous Hemodiafiltration (CVVHDF) and Recombinant Urate Oxidase to Treat Spontaneous Tumor Lysis Syndrome with Profound Hyperuricemia and Acute Kidney Injury}

E.Y. Park, R.L. Shim

Division of Nephrology, The Ohio State University, Columbus, OH, USA

Objective/Purpose: Tumor lysis syndrome (TLS) is a serious oncologic disorder characterized by severe electrolyte derangements and acute kidney injury which can occur spontaneously in rare cases or following cancer treatment. Presently there are no reports in treating spontaneous TLS (STLS) with concomitant use of continuous renal replacement therapy (CRRT) and urate oxidase. We describe the following case of STLS treated with CVVHDF and recombinant urate oxidase.

Case: A 44 year old male presented with two week complaints of fatigue, fever, and dyspnea. Physical examination was noted for cervical lymphadenopathy and truncal macular papular rash. Serum chemistries were as follows: sodium $132 \mathrm{mmol} / \mathrm{dL}$, potassium $6.7 \mathrm{mmol} / \mathrm{L}$, chloride $99 \mathrm{mmol} / \mathrm{dL}$, bicarbonate $7 \mathrm{mmol} / \mathrm{dL}$, bun $134 \mathrm{mg} / \mathrm{dL}$, creatinine $9.93 \mathrm{mg} / \mathrm{dL}$, uric acid $34.8 \mathrm{mg} / \mathrm{dL}$, phosphorous $9.1 \mathrm{mg} / \mathrm{dL}, \mathrm{LDH}$ $2800 \mathrm{U} / \mathrm{L}$. White blood cell count was $32.7 \mathrm{~K} / \mathrm{uL}$ (59\% mononuclear cells). Working diagnosis at this point was lymphoma with associated spontaneous TLS. Due to multiple metabolic abnormalities, uremia, and oliguria CVVHDF was the modality of renal replacement therapy. For profound hyperuricemia, rasburicase (recombinant urate oxidase) was also administered. Dialysate flow rate of $1500 \mathrm{ml} / \mathrm{hr}$ of the $4 \mathrm{~K} / 2.5$ Ca Prismasate ${ }^{\circledR}$ and replacement fluid of sterile water with $150 \mathrm{meq}$ of sodium bicarbonate at $1000 \mathrm{ml} / \mathrm{hr}$ were our CVVHDF parameters. Serum uric acid had fallen dramatically from $34.8 \mathrm{mg} / \mathrm{dL}$ to $6.4 \mathrm{mg} /$ $\mathrm{dL}$ and serum phosphorous dropped from $9.1 \mathrm{mg} / \mathrm{dL}$ to $5.5 \mathrm{mg} / \mathrm{dL}$ after 24 hours of intervention. His renal function recovered fully with a serum creatinine $0.8 \mathrm{mg} / \mathrm{dL}$ at discharge.

Discussion: Tumor lysis syndrome (TLS) is an oncologic emergency often seen in patients with lymphoproliferative disorders soon after treatment. Although rare, the spontaneous form of TLS may occur (STLS). In recent years urate oxidizers have been studied and used for TLS. CVVHDF is a modality which can prevent metabolic derangement rebounds during STLS versus intermittent hemodialysis. It may also be suggested that profound elevations of uric acid complicated by renal failure in STLS, a urate oxidizer may be indicated to prevent further tubular toxicity from urate crystallization and enhance renal recovery in adjunct to CVVHDF. It is presumed with such marked values of uric acid, enormous tumor burden is volatile and other electrolyte levels may potentially be lethal. The use of CVVHDF in addition to urate oxidase in STLS with substantial elevations of uric acid, hyperkalemia, and renal failure should be considered.
46

\section{CVVH vs SLED for Treatment of Critically III ARF Patients}

\author{
A.S. Okasha1, A. G. Adam¹, A.F. Elkoraei"1, A.A. Aglan¹, \\ H.M. Elkady ${ }^{2}$ \\ ${ }^{1}$ Faculty of Medicine, Alexandria University, Alexandria, \\ EGYPT, ${ }^{2}$ ICU Ddepartment, Astoon Hospital, Alkhobar, \\ AISharqiya, Saudi Arabia
}

Acute renal failure (ARF) is a common complication of critical illness.

The aim of this work was to compare renal recovery \& patient survival in critically ill patients developing ARF using 2 modalities of continuous renal replacement therapy (CRRT) which were continuous venovenous hemofiltration $(\mathrm{CVVH})$ and slow extended daily dialysis (SLED) and to evaluate the prognostic value of serum Cystatin $\mathrm{C}$ in patients with ARF.

This study was conducted on 20 adult patients admitted to critical care department at Alexandria Main University Hospital developing any of the following:

- Acute oliguric or anuric renal failure

- Rapidly rising serum creatinine level

- These conditions were unresponsive to medical management.

- These patients were prospectively divided into 2 groups; Group A (including 10 patients, CVVH session(s) were arranged for them), and Group B (including 10 patients, SLED session(s) were arranged for them).

During CRRT, each patient was subjected to measurement of central venous pressure, hourly urine output, and other relevant laboratory investigations.

Patient outcome was assessed for: renal recovery, restoration of adequate dieresis, or survival.

In SLED group there was significant increase in mean arterial pressure after CRRT than before CRRT.

Creatinine decreased significantly during therapy than before therapy in SLED group and also it significantly decreased at end of therapy in both groups and it significantly decreased at end of therapy than during therapy in both groups. Blood urea nitrogen significantly decreased in CVVH group during therapy than before therapy and also it significantly decreased in both groups at the end of therapy and 12 hours after the end of therapy than before CRRT, also it significantly decreased at end of therapy than during therapy in both groups, and it continued to significantly decrease 12 hours after CRRT than at end of CRRT in SLED group.

$\mathrm{pH}$ value has increased in SLED group between the end of CRRT than during CRRT, also there was significant increase at end of CRRT and 12 hours after CRRT than before CRRT.

Serum Cystatin C measurement didn't show intergroup difference at the time of inclusion in the study. At the end of CRRT, in CVVH group, it decreased than before CRRT, while in SLED group it was significantly higher than CVVH group, but it wasn't significantly different than before CRRT. 


\section{7 \\ Effect of Continuous Renal Replacement Therapy on Acute Kidney Injury after Open Heart Surgery and Application of AKICS Scoring System to those Patients}

\author{
J. Son, J. Kang, B.Y. Yang, E.Y. Seong, S.H. Song, D.W. Lee, \\ I.S. Kwak \\ Department of Internal Medicine, Pusan National \\ University Hospital, Busan, Republic of Korea
}

Acute kidney injury (AKI) after open heart surgery (OHS) was reported as 7.9 times higher mortality in hemodialysis patients. Continuous renal replacement therapy (CRRT) has been regarded as an effective modality to maintain the hemodynamic stability of the patients who were indicated to take RRT. To investigate the efficacy of CRRT, we compared the outcomes between CRRT-treated group and control group treated with fluid and diuretics. We also measured the AKICS score and Higgins score of both groups as prognostic markers. The thirty eight patients who had AKI after OHS were enrolled: CRRT group $(n=17)$, Control group $(n=27)$, respectively. We retrospectively analyzed the medical records such as pre-operative serum creatinine, duration of the surgery, central venous pressure, length of stay in ICU and time to return to the basal creatinine level. The CRRT group had higher APACHE III score $(\mathrm{p}<0.05)$ and poorer mortality $(\mathrm{p}<0.05)$ than control group. But the time to return to basal creatinine level was shorter in CRRT group compared to control group (p $<0.05)$. AKICS score over 12 showed significantly higher mortality in both groups ( $\mathrm{p}<0.05)$, but Higgins score did not showed significant differences. In summary, the renal recovery time could be shortened by CRRT despite the poorer basal characteristics of CRRT group. The AKICS scoring system might be a useful prognostic tool to predict mortality of the patients with AKI after OHS.

\section{8 \\ Hemodialysis during Acute Trauma Resuscitation: A Case Report}

\section{R. Buterakos, W.M. Bowling, K.A. Kralovich}

Department of Trauma Services, Hurley Medical Center, Flint, MI, USA

Background: The severity of the acidosis and the failure to correct quickly are both predictors of mortality in trauma. Here we report the case of a patient with intractable acidosis despite adequate resuscitation.

Case: 52 year-old man struck by a motor vehicle with an ISS 34. His initial systolic blood pressure was $40 \mathrm{mmHg}$, Glascow Coma Scale of 3 , and agonal respirations. Initial $\mathrm{pH}$ was 6.5 with a base-balance of $-24 \mathrm{mMol} / \mathrm{L}(-11$ correcting for chloride 126). Other labs were as follows: BUN $17 \mathrm{mg} / \mathrm{dL}$ and Creatinine $1.1 \mathrm{mg} / \mathrm{dL}$. The patient had developed acute abdominal compartment syndrome and underwent emergent decompressive laparotomy and vacuum dressing placement over the open abdomen. The abdomen drained 4,400 cc prior to dialysis. The patient received the following fluids for resuscitation: 31,850 cc crystalloids, 11 units PRBCs, 6 units FFP, and 1unit of cryoprecipitate. The base balance the time of dialysis was $-16 \mathrm{mMol} / \mathrm{L}(-13$ correcting for chloride 117) with a central venous pressure between 15 and $18 \mathrm{mmHg}$. The patient was hemodialyzed within 18 hours of arrival for persistent acidosis. Post-dialysis, the acidosis was reversed and the vasopressors were stopped. The base balance after hemodialysis was -5 ( -2 correcting for chloride 115$)$.

Conclusion: There are several potential mechanisms whereby early hemodialysis may be beneficial in trauma besides the correction of the acidosis. We believe this technique warrants further investigation.

\section{9 \\ Renal-Replacement Therapy Prevents Acute Kidney Injury: Case Report of a Neonate with Severe Gentamycin Toxicity}

\section{D.J. Askenazi \\ UAB Pediatric Nephrology, Birmingham, AL, USA}

Introduction: Gentamycin is a commonly used aminoglycoside antibiotic with known renal and ototoxicity. Whether toxicity is caused by elevated levels or duration of therapy is debatable.

Case: A 1 day old AA male accidentally received his mother's prescribed dose of intravenous gentamycin which amounted to 59 $\mathrm{mg} / \mathrm{kg}$ (about 30 usual neonatal dose). Gentamycin level $1.6 \mathrm{hrs}$ after dose confirmed severe gentamycin toxicity $(121.6 \mathrm{mg} / \mathrm{dl}$ - normal peak $10 \mathrm{mg} / \mathrm{dl}$ ) (see figure, p. 294). The nephrology team was consulted in anticipation of severe nephrotoxicity. Based on pharmacology properties, neonatal volumes of distribution $0.4-0.5 \mathrm{~L} / \mathrm{kg}$ and negligible protein binding, an $8 \mathrm{~F}$ hemodialysis catheter was placed in the right internal jugular vein for planned hemodialysis. In order to prevent toxic drug exposure hemodialysis was started 9.3 hours after initial gentamycin dose with blood flow of $50 \mathrm{ml} / \mathrm{min}$ and dialysis flow $=600 \mathrm{ml} / \mathrm{min}$. After 6.2 hours of dialysis gentamycin level was $9.1 \mathrm{mg} / \mathrm{dl}$ and dialysis was stopped.

Results: The half-life of gentamycin elimination (T1/2) was 5.8 hours before dialysis was initiated, $2.8 \mathrm{hrs}$ during dialysis and 8.4 hours after dialysis. A small rebound was noted shortly after dialysis. Serum creatinine ( $\mathrm{SCr}$ ) measured prior to dialysis was $0.8 \mathrm{mg} / \mathrm{dl}$ (representative of maternal creatinine). After dialysis, $\mathrm{SCr}$ was 0.5 $\mathrm{mg} / \mathrm{dl}$ and remained as such for the remainder of the hospitalization. Eletrolytes were acceptable and urine output remained brisk without intervention during the entire first week of life. The infant passed peripheral hearing screen at 2 weeks of life.

Conclusion: Hemodialysis effectively enhanced gentamycin removal. Rapid elimination of this drug likely avoided consequence of AKI and ototoxicity and suggests that peak levels alone do not determine toxicity. 


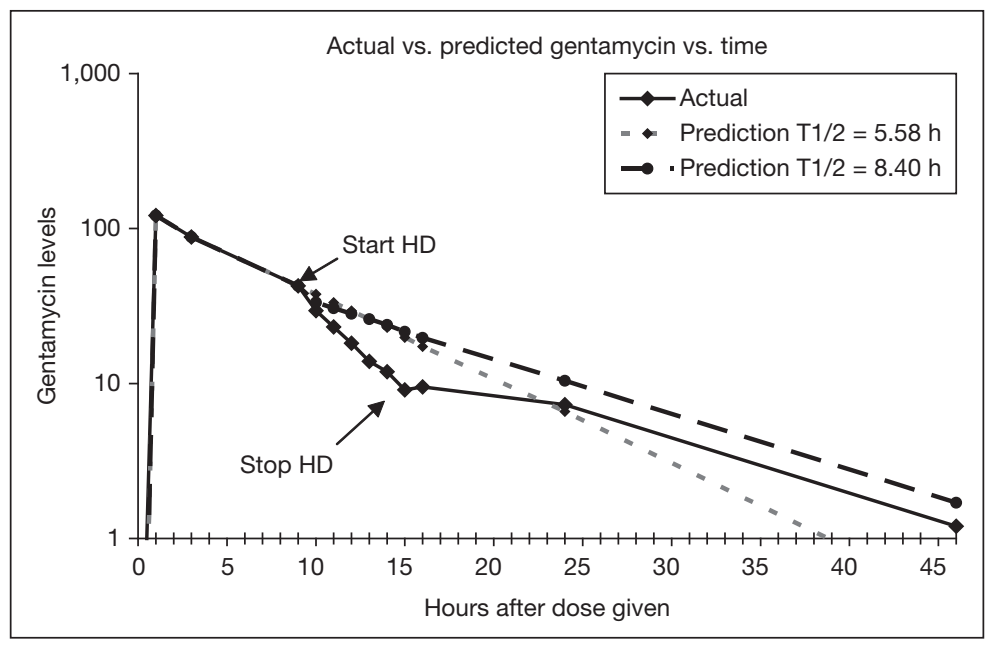

Figure for abstract 49

50

\section{Pediatric ARDS: Cytokine Assay and Cytokine Clearance by Hemofiltration}

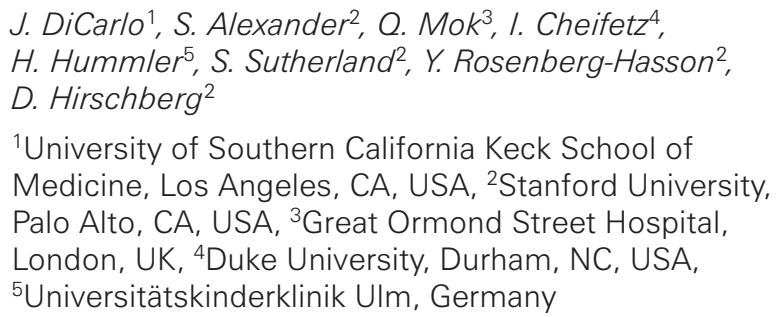

Methods: Six children with the acute respiratory distress syndrome (ARDS) following bone marrow transplantation had continuous hemofiltration initiated within 24 hours of fulfilling accepted criteria for ARDS. The children weighed between 8 and $70 \mathrm{~kg}$. The dose of ultrafiltration was $35+/-17 \mathrm{~mL} / \mathrm{kg} / \mathrm{hr}$. A multiplex assay of cytokines and chemokines was performed on serum and ultrafiltrate daily for three days, employing a Luminex BioPlex-HTX array analyzer to read color-coded microsphere beads, each coated with a specific reagent.

Results: The assay identified high serum concentrations of RANTES (4180 \pm 1034 picograms $/ \mathrm{mL})$, interferon-inducible protein-10 (IP-10 or CXCL10; $3688 \pm 3230 \mathrm{pcg} / \mathrm{mL}$ ), macrophage inflammatory protein 1-beta (MIP-1-beta; $1126 \pm 725 \mathrm{pcg} / \mathrm{mL}$ ), interleukin-8 $(566 \pm 1243 \mathrm{pcg} / \mathrm{mL})$ and IL-6 $(430 \pm 456 \mathrm{pcg} / \mathrm{mL})$. Moderate elevations were detected of vascular endothelial cell growth factor (VEGF), eotaxin, fibroblast growth factor 2 basic (FGFb), IL-1 $\beta$, IL-4, and IL-17. Nearly absent from serum were tumor necrosis factor-alpha, interferon-gamma, IL-2, IL-5, IL-13, and leptin.

Ultrafiltrate contained proportional concentrations of IP-10, MIP1-beta, IL-4, IL-8, IL-10 and IL-17, and moderate concentrations of VEGF, eotaxin and IL-6. Clearance was most efficient for IL-17 (3.3 $\pm 0.5 \mathrm{Liters} / \mathrm{kg} /$ day $),$ FGFb $(2.2 \pm 0.2 \mathrm{~L} / \mathrm{kg} / \mathrm{d}), \mathrm{IL}-8(1.9 \pm 1.0 \mathrm{~L} / \mathrm{kg} / \mathrm{d})$, IL-4, IL-1-beta, and IP-10.

Complete clearance data will be displayed in the poster session.

Discussion: Animal models of respiratory failure after bone marrow transplantation have established a timeline for cytokine production, with early predominance of interferon- $\gamma$, IL-2 and IL-6 followed by IL-8 and TNF-alpha. We demonstrate in humans the predominance of RANTES, IP-10 (CXCL10), MIP-1-beta, IL-6, and IL-8 at the onset of ARDS several weeks after transplantation. Notably, by this time TNF-alpha and IFN-gamma are absent. Hemofiltration might efficiently clear IL-17, FGFb, IL-8, IL-4, IL-1-beta and IP-10, and to a lesser extent IL-6. Hemofiltration seems ineffective for clearance of platelet-derived growth factor BB (PDGFBB) and RANTES. Granulocyte colony stimulating factor (G-CSF) is a cytokine utilized as a therapeutic agent; it was elevated in serum of children who had received it, and it was cleared with moderate efficiency.

Our findings have relevance to the evolution of therapy for respiratory failure following transplantation, and for ARDS in general. First, if blockade of TNF-alpha is to be effective, it should probably be instituted in advance of respiratory failure. Second, hemofiltration might effectively clear several relevant cytokines. Third, a follow-on study should follow a larger cohort serially to establish the pattern and timing of cytokine increment (e.g., IP-10, RANTES, IL-6, MIP-1-beta, IL-8) and perhaps decrement (especially TNF-alpha, IFN-gamma). These markers may be candidates for development of a clinically relevant battery identifying the patient in need of early intervention. 
patients develop AKI and become fluid overloaded (FO). We have previously shown worsening FO at CRRT initiation is independently associated with mortality risk in pediatric MODS pts. We wondered if mortality risk could be correlated with different FO thresholds which could then be used to develop guidelines regarding CRRT initiation.

Methods: Analysis was performed using the ppCRRT registry. All pts with MODS and FO at CRRT initiation who received CRRT for $>72$ hours were included. The relationship between mortality and FO severity at CRRT initiation (stratified into 3 groups: $<10 \%$, $10-20 \%$, and $>20 \% \mathrm{FO}$ ) was assessed by univariate and multivariate logistic regression.

Results: 174 patients were included (mortality 50\%). When stratified by degree of $\mathrm{FO},>20 \% \mathrm{FO}$ pts had higher mortality than $10 \%-20 \%$ FO or $<10 \%$ FO pts, $(p=0.01)$. ANOVA demonstrated significant differences between $\mathrm{FO}>20 \%$ and $\mathrm{FO}<10 \%$ pts in the number of inotropes administered, CVP, MAP, and age at CRRT initiation. Weight at CRRT initiation was significantly different amongst all three groups. There were no significant differences in PRISM II score at PICU admit, gender, CRRT modality, and eGFR. Univariate analysis demonstrated increased mortality odds ratio for $\mathrm{FO}>20 \%$ pts compared to $10-20 \% \mathrm{FO}(2.52)$ and $\mathrm{FO}<10 \%$ (2.43) pts. Multivariate analysis showed FO severity to be independently associated with increased mortality $(\mathrm{p}=0.003)$. Adjusted $\mathrm{OR}$ for $\mathrm{FO}>20 \%$ pts was 3.7 compared to $\mathrm{FO} 10-20 \%(\mathrm{p}=0.02) \mathrm{pts}$, and 10.3 compared to $\mathrm{FO}<10 \%$ pts $(\mathrm{p}<0.001)$.

Conclusions: Our ppCRRT data show: 1) Pediatric MODS pts with $>20 \%$ FO at CRRT initiation experienced significantly higher mortality than pts with lower levels of FO, despite no difference in PRISM II; 2) mortality risk increased incrementally with severity of FO; 3) survival was similar for pts with $<10 \%$ FO vs. $10-20 \%$ FO, suggesting a threshold near $20 \%$. While aggressive fluid resuscitation is often necessary in patients with MODS, we suggest that goal directed therapy protocols targeting CRRT initiation at $<20 \% \mathrm{FO}$, regardless of pt severity of illness, may improve outcomes in critically ill children who need CRRT.
52

\section{High Dose Continuous Veno-Venous Hemofiltration for Methotrexate Removal}

\author{
C.A Grafft, S. Markovic, J.C. Farmer, N. Leung \\ Mayo Clinic, Rochester, MN, USA
}

Introduction: Methotrexate (MTX) is a standard treatment for several malignant and autoimmune conditions and can be safety administered at high doses when given with urinary alkalinization and leucovorin rescue if renal function is adequate. However, MTX toxicity occurs with high serum drug concentration and prolonged exposure. Dialysis based methods of MTX removal have been ineffective because of the high protein binding of MTX and rebound with discontinued therapy. We report a case of high dose continuous veno-venous hemofiltration (CVVH) after charcoal hemofiltration to remove MTX.

Case Report: A 64 year old female with lymphoma received high-dose MTX after recovering from renal obstruction. Despite leucovorin rescue, urine alkalinization, and hydration to minimize toxicity; the 48 hour MTX level was elevated to $437.5 \mu \mathrm{mol} / \mathrm{L}$. The patient developed toxicity and serum creatinine increased to $2 \mathrm{mg} / \mathrm{dl}$. We treated this patient with 4 hours of charcoal hemoperfusion followed by $\mathrm{CVVH}$ at $5 \mathrm{~L} / \mathrm{hr}$ and blood flow rate of $300 \mathrm{ml} / \mathrm{minute}$. The substitution fluid was high bicarbonate containing and given 50\% pre and post dilution. After charcoal hemoperfusion, $1200 \mathrm{mg}$ of leucovorin was given followed by a $500 \mathrm{mg} / \mathrm{hr}$ maintenance infusion to compensate for CVVH removal. Additionally, our patient received diuretics to maintain high urine output and facilitate MTX removal. Within 12 hours of CVVH initiation, the serum MTX level was $106 \mu \mathrm{mol} / \mathrm{L}$ at which time the effluent methotrexate level was $57.7 \mu \mathrm{mol} / \mathrm{L}$. After 96 hours, the serum MTX decreased to $3.55 \mu \mathrm{mol} / \mathrm{L}$, but the rate of decline decreased and therefore charcoal hemoperfusion was repeated. Ultimately the MTX level decreased to $0.53 \mathrm{umol} / \mathrm{L}$ eleven days following CVVH initiation. Despite MTX removal with CVVH, our patient succumbed to her underlying disease and MTX toxicity.

Conclusion: High dose CVVH appeared effective at removing MTX at high serum levels; however, the efficacy decreased at low serum levels. Based on the kinetics, we hypothesize that protein binding may have affected the removal rate as saturation of the binding sites at high levels resulted in more free MTX and better clearance. Although removal was possible at lower serum levels, the efficacy decreased significantly.

Table for abstract 51

\begin{tabular}{|c|c|c|c|c|}
\hline & Group $1(<10 \%$ FO) & Group 2 (10-20\% FO) & Group 3 (>20\% FO) & p-value \\
\hline Mortality & $40.3 \%$ & $41.2 \%$ & $63 \%$ & 0.01 \\
\hline Age (years) & $11.5 \pm 6.9$ & $8.8 \pm 7.6$ & $6.2 \pm 6.2$ & $<0.001$ (1\&3 Only) \\
\hline Weight (kgs) & $48.7 \pm 32.4$ & $34.7 \pm 28.1$ & $20.9 \pm 18.4$ & $<0.001(1,2, \& 3)$ \\
\hline Gender & $\mathrm{M}=59.7 \%, \mathrm{~F}=40.3 \%$ & $\mathrm{M}=52.9 \%, \mathrm{~F}=47.1 \%$ & $\mathrm{M}=57.5 \%, \mathrm{~F}=42.5 \%$ & 0.81 \\
\hline PRISM II Score at ICU Admit & $13.0 \pm 6.6$ & $14.3 \pm 6.3$ & $16.1 \pm 9.1$ & 0.07 \\
\hline Inotrope Number at ICU Admit & $1.2 \pm 1.0$ & $1.4 \pm 1.1$ & $1.8 \pm 1.2$ & 0.01 (1\&3 Only) \\
\hline CVP at CRRT Initiation & $14.6 \pm 6.5$ & $16.9 \pm 6.7$ & $19.2 \pm 8.3$ & 0.01 (1\&3 Only) \\
\hline MAP at CRRT Initiation & $15.1 \pm 10.5$ & $19.4 \pm 8.9$ & $20.7 \pm 10.5$ & 0.01 (1\&3 Only) \\
\hline $\begin{array}{l}\text { eGFR at CRRT Initiation } \\
\left(\mathrm{mL} / \mathrm{min} / 1.73 \mathrm{~m}^{2}\right)\end{array}$ & $40.1 \pm 38.7$ & $41.1 \pm 32.8$ & $33.7 \pm 25.2$ & 0.40 \\
\hline CRRT Modality & $\begin{array}{l}\mathrm{CVVH}=25.4 \%, \\
\mathrm{CVVHD}=41.8 \%, \\
\mathrm{CVVHDF}=32.8 \%\end{array}$ & $\begin{array}{l}\mathrm{CVVH}=35.3 \%, \\
\mathrm{CVVHD}=47.1 \%, \\
\mathrm{CVVHDF}=17.6 \%\end{array}$ & $\begin{array}{l}\mathrm{CVVH}=26.0 \%, \\
\mathrm{CVVHD}=54.8 \%, \\
\mathrm{CVVHDF}=19.2 \%\end{array}$ & 0.23 \\
\hline
\end{tabular}

14th International Conference on Continuous

Blood Purif 2009;27:271-305

Renal Replacement Therapies (CRRT) 


\section{3 \\ Protocol for CRRT in the Patient with Elevated Intracranial Pressure}

\author{
D.W. Schmidt, B. Bean, T. Fulop, D. Hoekema, L. Juncos \\ Department of Nephrology, University of Mississippi \\ Health Care, Jackson, Mississippi, USA
}

In the management of increased intracranial pressure (ICP) there two principal therapeutic goals: 1. Maintain cerebral perfusion pressure 2. Decrease cerebral volume. In order to approach the second goal, increasing serum osmolarity is often desired (i.e. increased serum sodium to $145-155 \mathrm{meq} / \mathrm{dL}$ ). In patients with acute kidney injury and increased ICP, renal replacement therapy (RRT) can potentially compromise these therapeutic goals. Continuous RRT (CRRT) in this clinical setting offers several benefits including hemodynamic stability and precise control of metabolic and electrolyte balance. However commercially available replacement fluids currently contain a sodium concentration of $140 \mathrm{meq} / \mathrm{dL}$, compromising efforts maintain an elevated serum sodium. We have developed simple protocols for CRRT utilizing commercially available replacement fluids in conjunction with hypertonic saline that maintain elevated serum sodium during RRT. Our approach is illustrated in the case below.

A 19 year old white male was admitted with traumatic brain injury (TBI: basilar skull fracture, multiple frontal and temporal lobe contusions, and subarachnoid hemorrhage) from a motor vehicle collision. His ICP was initially elevated at $\sim 30 \mathrm{~cm} \mathrm{H} 2 \mathrm{O}$ and therefore received usual care and was initially stable. However, several days into his hospital course he developed life-threatening hyperkalemia and was consequently found to have severe rhabdomyolysis (CK >250,000 U/ $\mathrm{ml})$. In addition, his ICP had again increased. We therefore initiated continuous venovenous hemofiltration $(\mathrm{CVVH})$ for control of his hyperkalemia, removal of myoglobin and treatment of incipient renal failure (BUN <20). In order to maintain his serum sodium at 150 155 yet maximize myoglobin clearance, we employed CVVH using standard replacement fluid and an infusion of $3 \%$ saline. By titrating the ratio of replacement fluid to hypertonic saline we were able to affect a stable level of hypernatremia. Ultimately, the patient experienced a near complete recovery with little neuralgic sequelae and full recovery of his renal function. We have subsequently modified this approach so that the $3 \%$ saline is administered as a replacement fluid when conducting CVVHDF or CVVH with two replacement fluids.

Conclusions: A standardized CRRT protocol using commercial replacement fluids paired with $3 \%$ saline provides a simple method for delivering RRT while maintaining hypertonic plasma in the setting of increased ICP.

\section{4 \\ Early Intervention of CVVH for the Treatment of Acute Renal Failure following Multiple Bee Stings}

Zhang Ling, Tang Wan-xin, Fu Ping, Su Bai-hai, Qin Wei, Hu Zhang-Xue

Department of Nephrology, West China Hospital, Sichuan university, Chengdu, China

Objective: To investigate the effect of early intervention of $\mathrm{CVVH}$ on the treatment of acute renal failure following multiple bee stings.

Methods: 81 patients with bee stings injury during 19972007 were retrospectively analysis, in which 65 cases $(80.2 \%)$ suffered acute renal failure (ARF) and 60 cases (70.4\%) complicated with Multiple Organs Dysfunctional Syndrome (MODS). 64 cases with ARF were administered blood purification (APACHE Scores: 19.2 \pm 4.6 ):(1) CVVH/IHD group: early intervention of CVVH during 24-72h was performed, and then intermittent hemodialysis (IHD) was administered when conditions of patients were stable. (2) IHD group: IHD was performed three times per week. (3) PD group: Peritoneal dialysis was performed.

Results: (1)CVVH/IHD group: 19 cases(95\%) improved and discharged, but 1 case (5\%) died. (2) IHD Group: 30 cases $(78.9 \%)$ improved but 3 cases died (7.9\%), and 5 cases(13.2\%) withdrawer. (3)PD group: no patients improved, 2 cases (33.3\%) died, 3 cases $(50 \%)$ came to end stage renal disease (ESRD) and 1 case withdrawer. Survival and clinical improvement rates of CVVH/IHD and IHD groups are better than PD group. There was no significant differences of survival and clinical improvement rates between CVVH/IHD and IHD groups, but in the early stage, the extents of the decline in total bilirubin, alanine aminotransferase, creatine kinase and white blood are better in CVVH/IHD group than the IHD group $(\mathrm{P}<0.05)$. And adverse reactions (low blood pressure, arrhythmia) in the CVVH/IHD group were lower than the IHD group.

Conclusion: We believe that early CVVH treatment might be useful in patients with impaired organ function and shorten the hospitalization time, but whether can increase the survival rate of patients have yet to be confirmed by further studies. Peritoneal should not be used as the preferred treatment because of the poor effect. 
Future Trends

\section{5 \\ Continuous Renal Replacement Therapy in the Intensive Care Unit: Launch of a Program}

\author{
D.R. Deters, M.A. Rivera, H.P. Edgecomb, P.L. Violet, \\ M.S. Thies, E. Camarillo \\ Brooke Army Medical Center, Ft. Sam Houston, TX, USA
}

Background: Continuous Renal Replacement Therapy (CRRT) for patients in the Brooke Army Medical Center (BAMC) Critical Care Units was primarily provided through the inter-facility loan of CRRT trained nurses, from the co-located Institute of Surgical Research (ISR) Burn Intensive Care Unit (BICU). The BAMC hemodialysis department nursing staff also received training on CRRT using the NxStage machine. Although the utilization of CRRT is controversial and not a universally accepted treatment modality. However, early initiation of therapies such as CRRT in select patients has been suggested to be beneficial.

Objective: Identify the process for a successful implementation of an independent CRRT program in the BAMC Critical Care Units.

Main: The BAMC CRRT program was initially structured using the policy and procedures, as well as educational resources utilized in the ISR. Working with the assistance of the ISR Critical Care Nursing team to implement this program at BAMC, training materials as well as policies and procedures were updated and revised to develop a program tailored specifically to the care of the BAMC ICU patients. The patients in the BAMC ICU's would be provided CRRT using only the NxStage CRRT machine. The initial training for the ICU nursing staff was composed of a two day course; day one had four (4) hours of didactic instruction, followed by a 3 hour question and answer session related to the program, a review of the electronic charting, and program responsibilities. On the second day of the training, NxStage company vendors provided 4 hours of machine basics and a review of CRRT. Following the didactic portion, the group was divided into teams and assigned to a machine. This hand-on training provided each nurse the opportunity to learn how to set up the machine, perform exchange backs, and trouble shoot alarms. A final test was completed by each nurse on the NxStage machinery. The initial group of staff were labeled "Super-Users", with the understanding that they were to help facilitate the initiation of this program in the ICU, as well as assist in future staff training. The dialysis unit training involved 4 hours of hands on training by the NxStage company representative. Upon implementation, the dialysis unit staff will provide coverage for the CRRT treatment from 0800-1600 hrs, Monday through Friday.

Conclusion: Implementation of a new CRRT Program in a relatively CRRT novice ICU can be very daunting at times. The incorporation dialysis staff into the nursing care team activities provided its own challenges. However, with focused attention on teamwork and excellent administrative support, BAMC has successfully initiated a CRRT program in our ICU's.

\section{6}

\section{Adipose Tissue-Derived Stem and Regenerative Cells Ameliorate Ischemia- Reperfusion Induced Acute Kidney Injury}

\author{
Z. Feng, J. Ting, H.-C. Kuo, Z. Alfonso, J. Rutenberg, \\ B.M. Strem \\ Cytori Therapeutics, Inc., San Diego, CA, USA
}

Background: Acute Kidney Injury (AKI) represents a major clinical problem with high mortality and limited causal treatments. Preclinical studies have shown that cultured adipose derived cells are beneficial in Cisplatin-induced AKI, indicating a therapeutic potential of cell therapy. Uncultured Adipose Derived Stem and Regenerative Cells (ADRCs) are an easily accessible, abundant and heterogeneous population of cells, partially comprised of endothelial cells (and progenitors), smooth muscle cells, macrophages and mesenchymal-like stem cells. They can be isolated in large quantities by a minimally invasive liposuction and yield significantly higher quantities of progenitor cells per volume compared with bone marrow, making it an appealing source for autologous cell therapy.

Purpose: In this study, the effect of freshly isolated, uncultured, syngeneic ADRCs in a clinically relevant AKI model was investigated.

Methods: Twenty-nine adult male Fisher 344 rats were subjected to bilateral renal arterial and venous clamping for 38 minutes. Twenty minutes after reperfusion, animals received an intra-arterial infusion of $200 \mu \mathrm{l}$ of either vehicle (PBS, $\mathrm{n}=14)$ or 5 x 106 ADRCs $(\mathrm{n}=15)$. Serum creatinine (sCr) and blood urea nitrogen (BUN) were monitored daily for 7 days after AKI, and survival rates were recorded. In an additional experiment (ADRC, $n=3$; PBS, $n=3$ ), semi-quantitative histologic analysis of cast formation, a measure of tubular injury, was performed on day 3 after AKI.

Result: ADRCs could be safely administered intra-arterially in all animals. The $\mathrm{sCr}$ and $\mathrm{BUN}$ levels in the ADRC group were significantly reduced on days 1 through 6 after AKI as compared to those in the control group, with the largest difference on day3 (sCr: $3.03 \pm 1.58 \mathrm{mg} / \mathrm{dl}$ vs. $7.37 \pm 2.32 \mathrm{mg} / \mathrm{dl}, \mathrm{p}<0.0001$; BUN: $144.6 \pm 60.48 \mathrm{mg} / \mathrm{dl}$ vs. $296 \pm 76.74 \mathrm{mg} / \mathrm{dl}, \mathrm{p}<0.0001)$. Importantly, no ADRC-treated animal died during the 7 day follow-up, whereas $43 \%$ of animals in the control group died $(\mathrm{p}=0.005)$. Histologic analysis demonstrated that ADRC treatment significantly reduced tubular injury, indicated by far fewer intra-tubular cast formations $(<25 \%$ tubules vs. $>75 \%$ tubules, $\mathrm{p}<0.0001$ ).

Conclusion: This study shows that administration of syngeneic ADRCs dramatically reduces renal damage as well as mortality after ischemia/reperfusion injury. These results provide substantial evidence for further investigation of the potential of ADRC therapy for treating AKI. 
57

\section{Introducing New CRRT Technology with a Superuser Educational Model}

\section{C.D. Pierce, S. Strowjas}

Department of Intensive Care Unit, Brigham and Women's Hospital, Boston, MA, USA

Abstract: A large academic medical center was faced with the challenge of introducing new CRRT technology to replace its 10 year old devices. This initiative would require extensive education and clinical support of approximately $80 \%$ of the ICU nursing staff performing CRRT in 6 critical care units.

Methods Used: The educational model selected by our institution was to develop unit based superusers from all shifts to be available to their peers as clinical supports as well as to other units. "Superusers" were selected based upon their experiential knowledge, competency and motivational level. An extensive "hands on" training schedule was formulated in collaboration with the vendor's nurse educators to conduct 16 hour superuser sessions and 4 hour user classes. Extensive cooperation was required by the nurse managers for scheduling staff to attend the sessions in order to achieve the targeted goal.

Summary of Results: Using this "blitz" approach a total of 36 superusers were developed and 378 staff attended the user session during a three week period. A total of 2 superuser and 60 user sessions were conducted. Clinical support $24 / 7$ post training was provided by the vendor nurse educators, in addition to the hospital's superusers and clinical educators, while the technology was introduced into practice for 2 weeks.

Conclusions: Utilization of unit based superusers was a innovative and successful approach for providing access to additional clinical resources during the implementation to the new CRRT technology. Suggested improvements to this educational model would be to review patient assignments of the superusers to facilitate increased clinical availability to their peers. In addition, providing clinical educator support for off shift and weekend by beeper would provide an additional clinical resource during the transition period after vendor support had ended.

\section{8}

\section{Utilization of Renal Replacement Therapy for Critically III Patients}

F. Lai $^{1}$, R. Mehta ${ }^{2}$

${ }^{1}$ UCSD Department of Internal Medicine, San Diego, CA, USA, ${ }^{2}$ UCSD Department of Nephrology, San Diego, CA, USA

Acute kidney injury (AKI) affects up to $35 \%$ of critically ill patients in the hospital, with 5-6\% requiring renal replacement therapy (RRT). However, there is minimal information on the dialysis techniques utilized to treat AKI in the USA. A survey of National Kidney Foundation (NKF) members in 1995 by Mehta et al showed that intermittent hemodialysis (IHD) is the most common modality used for treatment of AKI, followed by continuous renal replacement therapy (CRRT) and finally peritoneal dialysis (PD) and sus- tained low-efficiency dialysis (SLED). Furthermore, there are no standardized tools to assess the delivery of dialysis. In this study, we conducted a similar survey to ascertain current practice patterns for ICU patients and assess for changes over the past decade. A 65-question survey was sent to NKF and Society of Critical Care Medicine (SCCM) members. Results showed that there was no difference among all the respondents (private practice nephrologists, academic nephrologists, and intensivists) in the dialysis modality used to treat AKI in the ICU setting. IHD is the most common modality in 50\% of the cases, followed by CRRT (40\%) and PD or SLED (10\%). The main reasons that IHD was used over CRRT or PD was that most feel IHD is effective $(47 \%)$, is routine procedure $(36 \%)$, and only a small fraction due to lack of experience with CRRT (2\%). Although $89 \%$ agree that CRRT is an acceptable alternative to IHD in the ICU setting, the major barrier for adopting CRRT is the lack of evidence on outcome. These prevailing thoughts have not changed from 1995, and the distribution of patients receiving CRRT and IHD modalities used are similar. Intensivists are less interested in outcome studies than the lack of clear indications for CRRT and standardized dosing protocols. In this snapshot of the current management practices for AKI, it is clear that we need clearer definitions on the diagnosis of AKI, when to initiate dialysis, and provide standardized protocols for IHD and CRRT modalities. Furthermore, outcome studies are very important in the adaptation of alternate therapies. Hopefully, this information will provide the foundation for further research in this area.

\section{9 \\ Phosphate-Containing Solution for Dialysis Prevents Hypophosphatemia during Continuous Renal Replacement Therapy}

M.E. Broman1, G.Z.V. Godaly², O. Carlsson², H. Friberg1, A. Wieslander ${ }^{2}$

${ }^{1}$ Department of Anaesthesiology and Intensive Care, Lund University Hospital, Lund, Sweden, ${ }^{2}$ Therapeutic Fluid Research, Gambro R\&D, Lund, Sweden

Purpose: The development of electrolyte disturbances in intensive care patients could be prevented by the use of better adapted dialysis fluids. A common problem is hypophosphatemia which has been shown to occur in up to $80 \%$ of the patients. Correction by intravenous phosphate supplementation is known to improve respiratory muscles, cardiac index, oxygen delivery to tissues and insulin resistance. Lately it has been reported that phosphate can be added directly to the dialysis fluid. This facilitates phosphate handling, but there is a risk of precipitation with calcium. An additional problem is that the amount of phosphate required to correct total body deficit varies and repeated serum measurements are needed to establish phosphate insufficiency. The process is time consuming and leads to treatment delay and excessive cost. This study evaluated the possibility to achieve and maintain normal phosphate balance over time by using a new phosphate-containing solution for dialysis.

Methods: Between January 2007 and July 2008, 42 critically ill patients with acute renal failure treated with continuous renal replacement therapy (CRRT), were enrolled in the study. We tested a new dialysis solution with $1.2 \mathrm{mM}$ phosphate, and compared it with standard medical treatment (Hemosol B0) in order to evaluate if we could 
maintain serum phosphate stability throughout the CRRT period. Patients were divided into three groups; group $1(n=14)$ receiving standard medical treatment and intravenous phosphate supplementation as required, group $2(\mathrm{n}=14)$ receiving the phosphate-containing solution as both dialysate and replacement solutions, and group 3 $(\mathrm{n}=14)$ receiving the phosphate solution as dialysate solution and Hemosol B0 as replacement solution.

Results: Standard medical treatment resulted in hypophosphatemia in $79 \%$ of the patients in group 1 . Patients in group 2 and 3 experienced stable serum phosphate levels throughout the study. However, the simultaneous intake of nutritional phosphate resulted in a slight increase in serum phosphate in group 2, while $13 \%$ in group 3 experienced one episode of hypophosphatemia. Ionized calcium, potassium, magnesium, $\mathrm{pH}, \mathrm{pCO}_{2}$ and bicarbonate remained unchanged throughout the study.

Conclusions: The new phosphate-containing solution for dialysis is safe, reduces the variability of serum phosphate levels during CRRT and reduces the incidence of hypophospatemia.

\section{0 \\ Pulse Pressure Variation Predicts Poor Hemodynamic Tolerance to Hemodialysis \\ R.H. Passos 1,2 , M.B. Ferri ${ }^{1,2}$, M.G. Pereira ${ }^{1}$, E. Figueiredo ${ }^{2}$, N. Akamine ${ }^{2}$, M.C. Batista ${ }^{2}$, O. Pavão ${ }^{2}$ \\ ${ }^{1}$ Hospital Rim e Hipertensão, ${ }^{2}$ Hospital Israelita Albert Einstein}

Background: Renal replacement therapy is frequently required in critically ill patients with acute kidney injury. With intermittent hemodialysis, large volumes of fluid are often removed over a relatively short period of time. This may jeopardize hemodynamic stability and induce tissue hypoxia and hypoperfusion. Pulse pressure variation (PPV) is increasingly used to guide fluid therapy in critical care, but its value to monitor fluid removal during hemodialysis is not known.

Objective: We designed the present study to investigate the effects of hemodialysis on mean arterial pressure (MAP) and marker of tissue oxygenation/perfusion $\left(\mathrm{ScvO}_{2}, \mathrm{CO}_{2}\right.$ gap, lactate) according to baseline PPV values.

Methods: The following hemodynamic parameters and lactate levels were collected just before and at the end of hemodialysis in mechanically ventilated patients: $\mathrm{ScvO} 2, \mathrm{CO} 2$ gap, and PPV. MAP was measured during hemodialysis and hypotensive episodes were defined as MAP $<65 \mathrm{mmHg}$.

\begin{tabular}{lllll}
\hline & $\begin{array}{l}\text { Group } \mathrm{H} \\
\text { Before }\end{array}$ & $\begin{array}{l}\text { Group H } \\
\text { End }\end{array}$ & $\begin{array}{l}\text { Group L } \\
\text { Before }\end{array}$ & $\begin{array}{l}\text { Group L } \\
\text { End }\end{array}$ \\
\hline $\mathrm{PPV} \%$ & 14 & $20 *(+43 \%)$ & $8 * *$ & $13 *(+63 \%)$ \\
$\mathrm{MAP} \mathrm{mmHg}$ & 75 & $58^{*}(-23 \%)$ & 79 & $65 *(-18 \%)$ \\
$\mathrm{Lactate} \mathrm{mmol} / 1$ & 1,1 & $2.5 *(+127 \%)$ & $0.5 * *$ & $1 *(+100 \%)$ \\
$\mathrm{SvcO}_{2} \%$ & 82 & $59^{*}(-28 \%)$ & 84 & $71 *(-15 \%)$ \\
$\mathrm{CO}_{2} \mathrm{Gap} \mathrm{mmHg}$ & 3 & $6.2^{*}(+106 \%)$ & 2.5 & 2.7 \\
\hline
\end{tabular}

$* \mathrm{P}<0.05$ End vs Before, ** $\mathrm{p}<0.05 \mathrm{H}$ vs L

14th International Conference on Continuous

Renal Replacement Therapies (CRRT)
Results: We studied 52 patients. The mean dialysis duration was $5 \mathrm{~h}$, and an average of $3420 \mathrm{ml}$ of fluid was removed. Just before hemodialysis, PPV was $>13 \%$ in 30 patients (group H) and $<13 \%$ in 22 patients (group L). Results are shown in the table. Hypotensive episodes were more frequent in group $\mathrm{H}$ than in group L (152 vs 102 , $\mathrm{p}<0,01)$.

Conclusion: Hemodialysis is poorly tolerated in patients with a high PPV (> 13\%). PPV monitoring and optimization could be useful to guide fluid removal and avoid tissue hypoxia and hypoperfusion in mechanically ventilated patients undergoing hemodialysis.

\section{CRRT Research}

\section{1 \\ Long Term Renal Function in Patients After Acute Renal Failure Treated With Continuous Veno-Veno Hemofiltration}

\author{
R.R. Kura, W.B. Reeves \\ Department of Internal Medicine/Nephrology, Penn \\ State University Hershey Medical Center and College of \\ Medicine, Hershey, PA, USA
}

Background: Continuous Veno-venous hemodiafiltration (CVVHDF) is an effective mode of renal replacement therapy in patients admitted to ICU with hemodynamic instability and renal failure. The aim of our study was to predict the long term outcome of renal function in patients who were treated with CVVHDF during their ICU stay.

Methods: We retrospectively analyzed 159 patients, who, over a period of 18 months, were admitted to ICU and required CVVHDF. Of these, 102 patients died (64\%) during their ICU stay.

Results: Among the 57 survived patients, those who had organ transplants and were taking calcineurin inhibitors, had preexisting ESRD or who lost follow-up were excluded (45), leaving 12 patients for analysis. The Mean ICU stay was $20 \pm 17$ days.Among these, 2 patients had pre-existing diabetes $(16.6 \%), 5$ patients had hypertension (41.6\%) and 4 patients had hyperlipidemia (33.3\%). The serum creatinine at the time of discharge from the hospital was $1.04 \pm 0.38$. After 3 to 36 months of follow up the serum creatinine for the group was $1.08 \pm 0.35$. This was not statistically significant from the discharge creatinine. Older patients were found to have higher creatinine levels at the time of discharge $(\mathrm{p}=0.014)$.

Conclusions: In this small sample of patients, there was no effect of length of ICU stay and use of CVVHDF on renal function after discharge. Acute renal failure during ICU stay requiring renal replacement therapy does not necessarily pose a danger to kidney function over the long term. 


\section{2 \\ Measurement of Total Body Water Using Heat Pulse Technology}

\author{
K. Lau, T. Kamai, J. Hopmans \\ University of California, Davis, CA, USA
}

Background: Total body water information is an essential piece of information for prescribing optimal dose of dialysis. However, obtaining accurate body water content information, especially in patients with an ever changing fluid status, has been hampered by the lack of an accurate yet easy to perform clinical tool for measuring the total body water volume.

Hypothesis: We hypothesize that the near skin thermal properties (body heat capacity and thermal conductivity) of human skin are directly related to its water content. This will enable total body water content estimations by non-invasive measurements.

Purpose: To test the clinical feasibility of applying a novel heat pulse probe in measuring total body water.

Methods: Based on the heat pulse technology that has long been used in soil science, we constructed 2 new prototype probes (linear and concentric). After generating a short heat pulse (3-5 seconds) by the heater, corresponding to a heat flux of about $60 \mathrm{Wm}-1$, the temperature responses of the skin are measured for 2-3 min by thermistor sensors built inside the probes.

Results: Using the probes, we have tested the heat pulse response on artificial medium. We have evaluated the preliminary results using a simple linear regression, relating the maximum temperature change in sponges with different volumetric water content. There was an excellent correlation for both probes. For the known thermistor accuracy of $0.01{ }^{\circ} \mathrm{C}$, sensitivities for water content are 1 and $1.5 \%$ for the concentric and linear probes, respectively. As water content is linearly correlated to heat capacity, this enables us to relate water content to HPP temperature responses.

Conclusions: The heat pulse probes described above have the potential to be a non-invasive tool to measure total body water content. Further testing on human subjects needed to confirm the clinical applicability of the probes in measuring body water content.
63

\section{Hypocalcemic Citrate Toxicity (HCCT) Associated with Regional Citrate Anticoagulation during Pediatric CRRT}

\author{
G.M. Fleming ${ }^{1}$, S. Walters ${ }^{2}$, T.A. Mottes ${ }^{2}$, P.D. Brophy ${ }^{3}$ \\ Department of Pediatrics, ${ }^{1}$ Vanderbilt University, \\ ${ }^{2}$ University of Michigan, ${ }^{3}$ University of lowa, Nashville, \\ Tennessee, USA
}

Background/Significance: Regional citrate anticoagulation for pediatric CRRT has gained in popularity, and studies in both children and adults have demonstrated improved filter life and safety over systemic heparinization. Citrate clearance during CRRT has been established to be equivalent to urea for both diffusion and convection. Some patients may have citrate accumulation due to a variety of factors such as citrate administration rate, CRRT clearance rate, and liver disease. With citrate accumulation ionized hypocalcaemia may develop with rising total calcium levels, which may lead to cardiovascular collapse. While measurement of citrate levels is possible, calcium monitoring is routinely available and practiced during CRRT. A ratio of total calcium to ionized calcium (Catot/Caion) has been found to closely correlate with total citrate levels. Hence, a Catot/ Caion ratio $\geq 2.5$ is suggestive of elevated citrate levels and represents risk for ionized hypocalcaemia.

The purpose of this study was to define the entity of Hypocalcemic Citrate Toxicity (HCCT) in a cohort of pediatric patients undergoing CRRT with regional citrate anticoagulation.

Methods: We performed a retrospective chart review of consecutive pediatric patients on CRRT in a single intensive care unit from 2005-2006. During this period, regional citrate anticoagulation was the only method used during CRRT without concomitant ECMO support. Demographic data, primary and secondary diagnosis, survival to PICU discharge, and calcium levels were recorded. Reason for RRT initiation was not collected for purposes of study. RRT was divided into 4 collection points, initiation then in 24 hour increments at $5 \mathrm{am}$ on RRT days 1-3. HCCT was stratified into three categories by Catot/ Caion ratio

Low Risk HCCT 1.73-2.1

Moderate Risk HCCT 2.11-2.49

High Risk $\quad$ HCCT $\geq 2.5$

Statistical analysis was limited to Fisher's Exact test comparing mortality for patients in the High Risk HCCT group with the total cohort.

Results: A total of 30 patients were identified for study. The mean age was $7.7+/-7$ years, mean weight $33.9+/-28 \mathrm{~kg}$, and mean BSA $0.98+/-0.67 \mathrm{~m} 2$. The recorded primary and secondary diagnosis was varied, however $12 / 30$ patients had sepsis, $3 / 30$ had liver disease, $1 / 30$ had liver transplantation, and $1 / 30$ had hyperammonemia.

Table for abstract 63

\begin{tabular}{lllll}
\hline & \multicolumn{4}{l}{ Data collection period } \\
HCCT group & Initiation CRRT & 24 hours CRRT & 48 hours CRRT & 72 hours CRRT \\
\hline High Risk & $2(6.7 \%)$ & $2(6.9 \%)$ & $1(4 \%)$ & $3(17.6 \%)$ \\
Moderate Risk & $19(63.3 \%)$ & $26(89.7 \%)$ & $22(88 \%)$ & $14(82.4 \%)$ \\
Low Risk & $9(30 \%)$ & $1(3.4 \%)$ & $2(8 \%)$ & $0(0 \%)$ \\
Total n & 30 & 29 & 25 & 17 \\
\hline
\end{tabular}


A total of 8 episodes of High Risk HCCT were recorded in 6 patients. Data for HCCT risk group by data collection period are presented in table 1 . Overall mortality was $36.7 \%$ in the cohort, and is presented in table 2 by maximum risk strata attained. Mortality was not significantly different for those patients with High Risk HCCT (maximum achieved) as compared to the cohort (Fisher's exact test $=0.6$ )

Conclusions: Using a Catot/Caion ratio, patients may be stratified by risk for hypocalcemic citrate toxicity. This study documents an incidence of High Risk HCCT from 4-17.6\% for each 24 hour period of study during pediatric CRRT. High Risk HCCT was not associated with an increased mortality. Further study will be required to define factors associated with increasing risk of HCCT.

\section{4 \\ Dose Separation at $20 \mathrm{ml} / \mathrm{kg} / \mathrm{hr}$ and $35 \mathrm{ml} / \mathrm{kg} /$ hr CVVHDF}

W. Fissell

Department of Biomedical Engineering, Cleveland Clinic, Cleveland, $\mathrm{OH}$, USA

Background: Several studies have attempted to compare different doses and modes of therapy in continuous renal replacement therapies in critically ill patients, and two recent trial showed no difference in outcomes between patients treated at $20 \mathrm{ml} / \mathrm{kg} / \mathrm{hr}$ and 35 $\mathrm{ml} / \mathrm{kg} /$ hour of continuous venovenous hemodiafiltration (CVVHDF). The nearly perfect identity of the Kaplan-Meier curves in the ATN Study suggests that despite excellent dose separation in urea clearance, dose separation might not have been achieved for some other clinically important solute.

Methods: We simulated $20 \mathrm{ml} / \mathrm{kg} /$ hour and $35 \mathrm{ml} / \mathrm{kg} / \mathrm{hour}$ CVVHDF using 4L of citrated bovine blood spiked with a polydiserse fluorescent tracer, FITC-Ficoll, a nd the Gambro Prismaflex system. Blood-side and dialyatse-side clearances of tracer molecules from 10-100kD were measured after 1 hour of therapy.

Results: Dialysate-side dose separation was excellent, with the higher-dose arm achieving approximately twofold higher clearance than the low-dose arm. Blood side clearances were nearly identical between the two arms, ranging form $4 \mathrm{ml} / \mathrm{min}$ at $10 \mathrm{kD}$ down to $2 \mathrm{ml} /$ $\min$ at $100 \mathrm{kD}$.

Discussion: Much middle molecule clearance in membranebased blood separation occurs through incorporation of macromolecules in a protein gel coat on the inner face of the dialyzer. Dialsyate-side clearance may not be an accurate estimate of solute removal from blood. Further simulations of continuous therapies appear warranted.

Table for abstract 65

\begin{tabular}{lllll}
\hline & Dalbavancin SC & Dalbavancin SC & $\begin{array}{l}\text { Dalbavancin CLtm } \\
(\mathrm{mL} / \mathrm{min})\end{array}$ & $\begin{array}{l}\text { Dalbavancin CLtm } \\
(\mathrm{mL} / \mathrm{min})\end{array}$ \\
\hline Hemodiafilter & M100 (n=5) & F160NR $(\mathrm{n}=5)$ & $\begin{array}{l}\text { M100 }(\mathrm{n}=5) \\
\text { Mean } \pm \text { SD }\end{array}$ & $\begin{array}{l}\text { F160NR }(\mathrm{n}=5) \\
\text { Mean } \pm \text { SD }\end{array}$ \\
Quf & Mean \pm SD & Mean \pm SD & $0.17 \pm 0.03$ \\
1 L/hr (16.6 mL/min) & $0.005 \pm 0.001$ & $0.010 \pm 0.002$ & $0.08 \pm 0.02$ & $0.32 \pm 0.05$ \\
3 $\mathrm{L} / \mathrm{hr}(33.3 \mathrm{hL} / \mathrm{min})$ & $0.005 \pm 0.001$ & $0.010 \pm 0.001$ & $0.17 \pm 0.04$ & $0.40 \pm 0.12$ \\
$6 \mathrm{~L} / \mathrm{hr}(100 \mathrm{~mL} / \mathrm{min})$ & $0.004 \pm 0.002$ & $0.008 \pm 0.002$ & $0.20 \pm 0.11$ & $0.98 \pm 0.30$ \\
\hline
\end{tabular}

\section{5 \\ Dalbavancin Transmembrane Clearance (CLtm) During Modeled Continuous Hemofiltration(CVVH)}

\author{
B.A. Mueller', K.H. Shah', J.H. Patel', D.D. DePestel', \\ A.M. Vilay ${ }^{1}$ \\ ${ }^{1}$ University of Michigan College of Pharmacy, Ann Arbor, \\ MI, USA, ${ }^{2}$ Department of Pharmacy and Pharmacology, \\ University of Bath, Bath, UK
}

Background: Dalbavancin is a lipoglycopeptide with antimicrobial activity against Gram-positive pathogens. It has a slow renal clearance (CLr) $(0.26 \mathrm{~mL} / \mathrm{min})$ enabling once weekly dosing. CVVH dalbavancin CLtm is unknown. The small volume of distribution (15.7-18.3 L) and molecular weight $(\sim 1800 \mathrm{Da})$ of dalbavancin suggests that it may be removed by CVVH. However, its extensive protein binding (93\%), makes it difficult to predict the overall extent of CVVH clearance.

Purpose: To determine dalbavancin CLtm during CVVH.

Methods: A validated in-vitro bovine blood-based CVVH model using AN69 (M100, Hospal) and polysulfone (F160NR, Fresenius) hemodiafilters was employed with ultrafiltration rates (Quf) of 1, 2, 3 , and $6 \mathrm{~L} / \mathrm{h}$. Blood flow (Qb)was varied between $200-400 \mathrm{~mL} / \mathrm{min}$ to maintain appropriate filtration fractions. Blood (A) and ultrafiltrate (UF) samples were obtained from the arterial and UF ports of the CVVH tubing at each Quf. Formed UF was recirculated back into the system, forming a closed circuit. Samples were assayed for dalbavancin using HPLC coupled with mass spectrometry. The lower limit of dalbavancin quantification was $0.05 \mathrm{ug} / \mathrm{mL}$ in ultrafiltrate and 1.00 $\mathrm{ug} / \mathrm{mL}$ in plasma. Samples were also assayed for urea, the control solute, by a kinetic test with urease and glutamate dehydrogenase coupled with absorbance photometry. The sieving coefficient (SC) and CLtm of dalbavancin and urea were then calculated using the following equations: $\mathrm{SC}=\mathrm{UF} / \mathrm{A} ; \mathrm{CLtm}=\mathrm{SC} * \mathrm{Quf}$.

Results: Urea SC was approximately 1 regardless of filter type and Quf. Dalbavancin SC with the F160NR filter is significantly larger than with the M-100 filter at the same Quf $(\mathrm{p}<0.05)$, however increased Quf did not affect SC ( $>>0.05)$ within each filter type.

Conclusion: Dalbavancin CLtm is relatively small but is similar to the CLr reported in healthy normal's. Consequently, CVVH may contribute to a shorter dalbavancin dosing interval particularly when the F160NR filter and higher Quf are employed. 
66

\section{Methotrexate Clearance During Three Continuous Renal Replacement Therapy (CRRT) Modalities}

D.J. Askenazi1, B.A. Mueller², H. Haines'3, J. Alten', A.M. Vilay ${ }^{2}$

${ }^{1}$ Department of Pediatrics, Division of Nephrology, University of Alabama at Birmingham, Birmingham, AL, USA, ${ }^{2}$ University of Michigan College of Pharmacy, Ann Arbor, MI, USA, ${ }^{3}$ Department of Pediatrics, Division of Hematology/Oncology, University of Alabama at Birmingham, Birmingham, AL, USA, ${ }^{4}$ Department of Pediatrics, Division of Critical Care, University of Alabama at Birmingham, Birmingham, AL, USA

Background: Methotrexate-induced renal dysfunction results in prolonged methotrexate exposure and increased risk of myelosuppression, mucositis, and hepatotoxicity. Several intermittent extracorporeal modalities have been used to enhance methotrexate clearance. However, in critically ill patients, continuous therapies may be preferable. The addition of albumin to dialysate during continuous venovenous hemodialysis (CVVHD), also known as single pass albumin dialysis (SPAD), has been reported to improve the clearance of methotrexate as well.

Purpose: Three different modalities of CRRT, including SPAD, were used to treat elevated methotrexate plasma concentrations in a critically ill 13 year old female with non-metastatic osteosarcoma. An analysis of methotrexate concentration data from plasma and effluent samples was performed to compare methotrexate clearances between these CRRT modalities.

Table for abstract 66

\begin{tabular}{llllll}
\hline Modality & Qd (mL/h) & Quf $(\mathrm{mL} / \mathrm{h})$ & Half-life $(\mathrm{h})$ & $\begin{array}{l}\text { Saturation } \\
\text { coefficient }\end{array}$ & $\begin{array}{l}\text { CLtm/ } \\
(\mathrm{mL} \text { min })\end{array}$ \\
\hline No RRT & - & - & 14.8 & - & - \\
CVVHD 1 & 2100 & 450 & 6.5 & 0.67 & 28.4 \\
SPAD 1 & 2100 & 450 & 7.4 & 0.86 & 36.5 \\
CVVHD 2 & 2100 & 450 & 12.4 & 0.81 & 34.5 \\
CVVHDF & 2000 & 2950 & 151.6 & 0.78 & 64.4 \\
SPAD 2 & 2100 & 450 & 49.5 & - & 36.5 \\
No RRT & - & - & 41.0 & - & - \\
\hline
\end{tabular}

Methods: SPAD was conducted with a $1.85 \%$ albumin dialysate concentration. Plasma and effluent samples were collected serially and assayed for methotrexate using fluorescence polarization immunoassay. Non-compartmental pharmacokinetic analysis was completed. Saturation coefficients (SA) for each continuous extracorporeal therapy were determined as the ratio of the effluent to plasma methotrexate concentrations.

Results: Blood flow rate during all continuous extracorporeal therapies was $180 \mathrm{~mL} / \mathrm{h}$. (Table Legend: $\mathrm{Qd}=$ dialysate flow rate; Quf=ultrafiltration production rate; $\mathrm{CLtm}=$ transmembrane clearance; $\mathrm{RRT}=$ renal replacement therapy; $\mathrm{CVVHDF}=$ continuous venovenous hemodiafiltration)

Conclusion: Methotrexate clearance related more to effluent rate than modality. The longer methotrexate half-life seen with CVVHDF was likely due to the effects of co-administered glucarpidase. The addition of albumin to dialysate did not appreciably enhance methotrexate clearance. In methotrexate intoxication cases requiring CRRT, high effluent rates should be employed rather than instituting SPAD to enhance methotrexate removal.

67

In vitro Evaluation of Mechanisms of Elimination and of the Therapeutic Levels of Piperacillin and Tazobactam During HighVolumes-CVVHDF

\author{
G. Dimita1, E. Salvatorelli2, M.C. Tirindelli3, G. Minotti², \\ F.E. Agròn ${ }^{1}$, M. Ferranninit
}

${ }^{1}$ Department of Anesthesia and Intensive Care, University Campus Bio-Medico, Rome, Italy, ${ }^{2}$ Center for Integrated

Research and Drug Sciences, University Campus

Bio-Medico of Rome, Rome, Italy, ${ }^{3}$ Department of Hematology, University Campus Bio-Medico of Rome, Rome, Italy, "Nephrology and Dialysis Unit - "Tor Vergata" University Hospital, Rome, Italy

Background: Piperacillin-tazobactam (Pip-Tzb) is a beta-lactam-beta-lactamase inhibitor combination with a broad spectrum of antibacterial activity against gram-positive as well as gram-negative pathogens and it is commonly used to treat infections in ICU patients.

Table for abstract 67

\begin{tabular}{lccccc}
\hline $\begin{array}{l}\text { Adsorption } \\
\text { time }\end{array}$ & Pip (microM) & Tzb (microM) & $\begin{array}{l}\text { HV-CVVHDF } \\
\text { time }\end{array}$ & Pip (microM) & Tzb (microM) \\
\hline 0 & \multicolumn{5}{c}{} \\
5 & 591.4 & 232.7 & 0 & 381 & 122.5 \\
15 & 478.9 & 121.2 & 5 & 301.3 & 85.2 \\
30 & 479.4 & 124.5 & 10 & 218.1 & 60.5 \\
45 & 466.1 & 117.7 & 20 & 54.23 & 11.04 \\
60 & 468.5 & 119.5 & 30 & 33.8 & 44.53 \\
90 & 452.1 & 118.7 & 40 & 23.9 & 2.27 \\
120 & 425.7 & 116.7 & 50 & 20.1 & 1.43 \\
& 416.8 & 117.7 & 60 & 19.8 & 0.64 \\
& & 90 & 19.9 & 0.56 \\
& & 120 & 19.8 & 0.56 \\
& & 240 & 18.9 & 0.35 \\
\hline
\end{tabular}


Controversial data have been published about the correct dosage during High-Volumes-CRRT. Nevertheless to obtain a therapeutic effect, Pip-Tzb serum levels must be continuously above the MinimalInhibitory-Concentration (MIC) level, 30-120microM and 35microM for Pip and Tzb respectively.

Aim: To evaluate the Pip-Tzb serum levels during an in vitro HV-CVVHDF using a AV1000 polysulfone filter; to investigate the adsorption as a mechanism of clearance.

Method: A single-pool model with 1 liter human fresh-frozenplasma (FFP) was employed, adding $67 \mathrm{mg}$ of Tzb and 320mg of Pip, to obtain its own maximum level of concentration (Cmax). To test adsorption, the FFP-Pip-Tzb solution was circulated at $150 \mathrm{ml} /$ min, without Qd, Qs and Uf, for 2 hours. Samples were obtained at start and every 15 minutes for the first hour, then every $30 \mathrm{~min}$. Then, HV-CVVHDF was started, with Qb $150 \mathrm{ml} / \mathrm{min}$, Qd $2000 \mathrm{ml} / \mathrm{h}$, Qs $2500 \mathrm{ml} / \mathrm{h}$ (according to $35 \mathrm{ml} / \mathrm{kg} / \mathrm{h}$ doses for a $70 \mathrm{~kg}$ weight patient). FFP-Pip-Tzb solution's samples were obtained starting and after 5, $10,20,30,40,50,60,90,120,240$ and 300 minutes of therapy. Drugs levels were measured using HPLC method.

Results: The Pip-Tzb levels during the first part of test decreased of $20 \%$ in five minutes and of $30 \%$ after 2 hours. During HV-CVVHDF, the Pip-Tzb levels were under the MIC after 1 hour of treatment.

Conclusion: Polysulfone fibers filter is able to adsorb a large amount of Pip-Tzb just in 5-10 minutes of treatment. Moreover the Pip-Tzb clearance is very high during HV-CVVHDF treatment, with levels lower than the MIC after the first hour of treatment. More investigations need to confirm these findings and perhaps to find new administration schemes.

\section{8}

\section{Stabilizing Tazocin ${ }^{\circledR}$ Plasma Levels in Septic Patients on CRRT}

\section{G. Dimita', E. Salvatorelli², M.C. Tirindelli , F.E. Agrò', M. Ferrannini ${ }^{4}$ \\ ${ }^{1}$ Department of Anesthesia and Intensive Care, University Campus Bio-Medico, Rome, Italy, ${ }^{2}$ Center for Integrated \\ Research and Drug Sciences, University Campus \\ Bio-Medico of Rome, Rome, Italy, ${ }^{3}$ Department of \\ Hematology, University Campus Bio-Medico of Rome, \\ Rome, Italy, "Nephrology and Dialysis Unit - "Tor Vergata" \\ University Hospital, Rome, Italy}

Tazocin ${ }^{\circledR}$ (piperacillin and tazobactam) is commonly used in ICU (Intensive Care Unit) for the treatment of infections. Controversial data have been published about the dosing regimen of Tazocin in septic patients undergoing CRRT treatments. In an accompanying simulation study we demonstrated that Tazocin ${ }^{\circledR}$ concentrations dropped below the MIC (Minimal Inhibitory Concentration) of piperacillin and tazobactam. The aim of this study was to establish if adding the reinfusion bags with appropriate amounts of piperacillin and tazobactam succeeded in stabilizing Tazocin ${ }^{\circledR}$ plasma levels at MIC during CRRT. This simulation study was done by using 1 liter of fresh-frozen plasma (FFP) and a flask as drug reservoir. The latter was added with piperacillin and tazobactam at their Cmax values $(259 \pm 81 \mathrm{mg} / \mathrm{L}$ for piperacillin and $27.9 \pm 7.67 \mathrm{mg} / \mathrm{L}$ for tazobactam). We used the dialyzer Fresenius Medical Care Multifiltrate, equipped with an $1.8 \mathrm{~m} 2$ polysulfone AV1000 filter, set in CVVHDF modality, with Qb $145 \mathrm{ml} /$ min, Qd 2000, Qs 2500, and UF of 0. Aliquots of the solution samples were taken from the flask at the times indicated in the Table, and were analyzed by HPLC with diode array spectroscopy. By assuming MIC ranges of $16-64 \mathrm{mg} / \mathrm{L}$ for piperacillin and $10 \mathrm{mg} / \mathrm{L}$ for Tazobactam, we prepared reinfusion bags added with $54 \mathrm{mg} / \mathrm{L}$ and $6.75 \mathrm{mg} / \mathrm{L}$ of the two drugs, respectively. On-filter adsorption of Tazocin ${ }^{\circledR}$ during active filtration was minimized by two hours preconditioning of the dialyzer without Qd, Qs and UF. After an initial reduction of drugs plasma levels attributable to filtration, concentrations of piperacillin and tazobactam plateaued above the corresponding MIC (see table).

Thus, the method may be useful to stabilize Tazocin ${ }^{\circledR}$ concentrations in septic patient undergoing CRRT and to set drugs plasma levels over therapeutic ranges in these patients. These findings call for clinical testing and new dosing regimens of Tazocin ${ }^{\circledR}$ in septic patients on CRRT.

\begin{tabular}{lll}
\hline Time (minutes) & Tazobactam $(\mathrm{mg} / \mathrm{L})$ & Piperacillin $(\mathrm{mg} / \mathrm{L})$ \\
\hline 0 & 44 & 290 \\
5 & 31.6 & 174 \\
10 & 23.7 & 135 \\
30 & 13.5 & 119 \\
60 & 10.2 & 96 \\
90 & 9.8 & 91 \\
120 & 9.6 & 88 \\
180 & 9.3 & 81 \\
\hline
\end{tabular}

69

\section{Successful Outcome Utilizing Continuous Veno-Venous Hemofiltration in Severe Crush Syndrome Complicated with Acute Pancreatitis after Wenchuan Earthquake}

\author{
Liu Fang, Zhong Hui, Zhang Ling, Su Baihai, Chen Xiaolei, \\ Liu Ling, Tao Ye, Fu Ping \\ Division of Nephrology, West China Hospital, Sichuan \\ University, Chengdu, China
}

Background: An earthquake took place in Wenchuan area, which was measured at 8.0 Richter scale. The case and the treatment of severe crush syndrome complicated with acute pancreatitis after the earthquake was seldom reported.

Case: We present a case of 46-year-old man experienced the Wenchuan earthquake and had been buried with his legs being crushed for 6 hours. 2 days later, he was admitted to Nephrology division and diagnosed with crush syndrome, traumatic rhabdomyolysis and acute renal failure (ARF). On the $3^{\text {rd }}$ hospitalized day, he complained of abdominal pain and distension with increased serum concentration of amylase and lipase. Abdominal computer tomography (CT) confirmed the diagnosis of acute pancreatitis.

Result: By continuous veno-venous hemofiltration (CVVH) and other comprehensive treatments, the patient recovered completely.

Conclusion: This is the first case of severe crush syndrome complicated with acute pancreatitis after the Wenchuan earthquake. CVVH combined with the comprehensive treatment should be beneficial in such patient. 


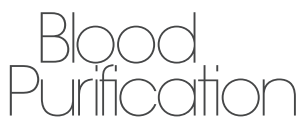

Numbers refer to abstract numbers

Abraham, G. 29

Adam, A.G. 46

Aglan, A.A. 46

Agrò, F.E. 67, 68

Akamine, N. 60

Alexander, S. 50

Alfonso, Z. 56

Alfurayh, O. 23

Al Mosawi, A. 3

Alten, J. 66

Ambalavanan, N. 5, 6

Ambikapathi, P. 29

Amdur, R.L. 27

Amigues, L. 15, 35

Amodeo, S. 27

Annich, G.M. 38

Askenazi, D. 8

Askenazi, D.J. 5, 6, 7, 49, 66

Attaluri, A. 33

Bagga, A. 22

Baihai, S. 21, 54, 69

Bakker, J. 31

Banaei-Kashani, K. 25

Barillo, D.J. 16

Batista, M.C. 60

Bean, B. 53

Blackbourne, L.H. 16

Bowling, W.M. 48

Britton, M.L. 14

Broman, M.E. 59

Brophy, P.D. 63

Buterakos, R. 48

Camarillo, E. 55

Canaud, B. 15

Carlsson, O. 59

Cartin-Ceba, R. 24, 25

Chaaban, M.H 23

Chakravarty, R. 10

Chan, C.-M. 34

Chang, M. 9

Chawla, L.S. 27

Cheifetz, I. 50

Chil Choi, Y. 4

Cho, Y. 12

Choi, A. 12

Chon, G.R. 4

Choong, H.-L. 34

Chua, A.N. 8

Chung, K.K. 16

Clark, E.G. 36

Clark, W. 33
Cosmin, A. 11

Cristol, J.P. 35

Cutter, G. 5, 6, 7

Dang, T. 37

Delabre, J.-P. 15, 35

Dennen, P.F. 17

DePestel, D.D. 65

Deters, D.R. 55

DiCarlo, J. 50

Dimita, G. 67, 68

Dimmitt, R.A. 5, 6, 7

Edgecomb, H.P. 55

Elkady, H.M. 46

Elkoraei, A.F. 46

Emmerich, J. 30

Fang, L. 18, 19, 69

Farmer, J.C. 52

Feng, Z. 56

Ferrannini, M. 67, 68

Ferri, M.B. 60

Figueiredo, E. 60

Finkel, K.W. 14, 37

Fissell, W. 64

Fleming, G.M. 63

Flores, F.X. 8

Folber, F. 2

Friberg, H. 59

Fu, P. 32

Fulop, T. 11, 53

Gajic, O. 25

George, S. 41

Geus, H.D.E. 31

Godaly, G.Z.V. 59

Goetze, A. 30

Goldstein, S.L. 8, 51

Grafft, C.A 52

Gruenert, J. 30

Gupta, A. 26

Gupta, V. 37

Hackbarth, R. 8

Haines, H. 66

Hamilton, K. 5, 6, 7

Han, S. 9

Hansen, C.A. 40

Hari, P. 22

Harikrishna 10

Haugen, E.N. 24

Hentschel, J. 39
Heung, M. 42, 44

Hickstein, H. 39

Hirschberg, D. 50

Hixon-Vermillion, B. 43

Ho Wang, J. 4

Hoekema, D. 53

Hong-yu, Q. 20

Hopmans, J. 62

Horie, A. 28

Hu, Z. 32

Huang, S. 32

Huang, Z. 33

Hui, Z. 69

Hummler, H. 50

Hwang, E. 9

Ince, C. 31

Jamieson, J. 44

Jaussent, A. 35

Jing, L. 21

Jonquet, O. 15

Juncos, L. 11, 53

Kakulavaram, A. 44

Kamai, T. 62

Kamaladevi, L.N. 29

Kamra, A.K. 41

Kang, J. 47

Kashani, K.B. 24

Kaul, A. 26

Kawasaki, T. 28

Keniston, A. 17

Kershaw, D.B. 38

Khan, M. 33

Khanna, P. 29

Kim, H. 9

Kim, J. 9

Kim, J.-H. 13

Kim, J.-K. 13

Kimmel, P.L. 27

King, B.T. 16

Kitayama, H. 28

Klammt, S. 39

Klouche, K. 15

Koball, S. 39

Koristek, Z. 2

Kralovich, K.A. 48

Kuo, H.-C. 56

Kura, R.R. 61

Kwak, I.S. 47

Lai, F. 58
Laney, D. 5, 6, 7

Lau, K. 62

Lee, W.-D. 13, 47

Lee, Y. 23

Letteri, J. 33

Leung, N. 52

Li, W. 18

Liang, J. 32

Ling, L. 69

Ling, Z. 54, 69

Lodha, R. 22

Lundy, J.B. 16

McAfee, N.G 40

Macnowski, J. 42

Mallikarjun 10

Mandal, S. 26

Markovic, S. 52

Mathew, M. 29

Mehrotra, A. 29

Mehta, P. 22

Mehta, R. 7, 58

Minotti, G. 67

Mitzner, S. 30

Mok, Q. 50

Mottes, T.A. 38, 63

Mount, K. 43

Mueller, B.A. 42, 65, 66

Navratil, M. 2

Noble, J.L.E. 31

Ognjen, G. 24

Okasha, A.S. 46

Palant, C.E. 27

Park, E.Y. 45

Park, J.M. 4

Park, S. 9

Parrish, J. 43

Passos, R.H. 60

Patel, J.H. 65

Pavão, O. 60

Peng, L. 1

Pereira, M.G. 60

Picot, M.C. 35

Pierce, C.D. 57

Ping, F. 18, 19, 20, 21, 54, 69

Podoll, A.S. 14, 37

Podymow, T. 36

Prasad, N. 26

Ramet, H. 35

\section{KARGER}

(c) 2009 S. Karger AG, Basel

Fax +4161306 1234

E-Mail karger@karger.ch

www.karger.com

Accessible online at:

www.karger.com/bpu 
Reddy, V. 10

Reeves, W.B. 61

Rivera, M.A. 55

Rosenberg-Hasson, Y. 50

Rutenberg, J. 56

Sachdeva, K.S. 37

Salvatorelli, E. 67, 68

Samavedam, S. 10

Schmidt, D.W. 53

Selby, M.G. 24

Seo, M. 12

Seong, E.Y. 47

Shah, K.H. 65

Sharma, R.K. 26

Shidham, G. 43

Shim, R.L. 45

Shin, H.J. 4

Sinha, A. 22
Smardova, L. 2

Son, J. 47

Song, S.H. 47

Songmin, H. 18, 20

Stange, J. 30, 39

Strem, B.M. 56

Strowjas, S. 57

Strube, S. 30, 39

Suh, J. 12

Sung, Y. 12

Sutherland, S. 50

Sutherland, S.M. 51

Symons, J. 40

Tan, C.-S. 34

Tan, H.-K. 34

Tao, Y. 32

Thies, M.S. 55

Ting, J. 56

Tirindelli, M.C. 67,68
Tomiska, M. 2

Tripathi, R. 26

Tu, Y. 32

Udeozo, O.I. 24

Uehara, M. 28

Ui Lee, T. 4

Vilay, A.M. 42, 65, 66

Violet, P.L. 55

Wada, N. 28

Wadehra, D. 41

Wadhwa, N.K. 41

Walcher, A.M. 17

Walters, S. 63

Walters, S.W. 38

Wan-xin, T. 54

Wei, Q. 19, 21, 54
White, C.E. 16

Wieslander, A. 59

Wolf, S.F. 16

Xiao, H. 1

Xiaolei, C. 19, 21, 69

Xuhua, M. 20, 21

Yamada, M. 28

Yang, B.Y. 47

Ye, T. 18, 19, 21, 69

Youjuan, W. 18

Zeng, X. 32

Zhangxue, H. 18, 19, 54

Zijlstra, F. 31

Zuo chuan, L.F. 20 